\title{
Comparative Transcriptome Analysis of Bombyx mori (Lepidoptera) Larval Hemolymph in Response to Autographa californica Nucleopolyhedrovirus in Differentially Resistant Strains
}

\author{
Xin-yi Ding ${ }^{1}$, Xue-yang Wang ${ }^{1,2}$, Yun-hui Kong ${ }^{1}{ }^{(\mathbb{D}}$, Chun-xiao Zhao ${ }^{1}$, Sheng Qin ${ }^{1,2}$, Xia Sun ${ }^{1,2}$ \\ and Mu-wang $\mathrm{Li}^{1,2, *(\mathbb{D})}$
}

1 Jiangsu Key Laboratory of Sericultural Biology and Biotechnology, School of Biotechnology, Jiangsu University of Science and Technology, Zhenjiang 212100, China; ding_xin_yi@163.com (X.-y.D.); xueyangwang@just.edu.cn (X.-y.W.); kongyunhui1@gmail.com (Y.-h.K.); www_zcx123456@163.com (C.-x.Z.); qinsheng@just.edu.cn (S.Q.); sunxia8428@163.com (X.S.)

2 Key Laboratory of Silkworm and Mulberry Genetic Improvement, Ministry of Agriculture and Rural Affairs, Sericultural Research Institute, Chinese Academy of Agricultural Science, Zhenjiang 212100, China

* Correspondence: mwli@just.edu.cn

check for updates

Citation: Ding, X.-y.; Wang, X.-y.;

Kong, Y.-h.; Zhao, C.-X.; Qin, S.; Sun,

X.; Li, M.-w. Comparative

Transcriptome Analysis of Bombyx

mori (Lepidoptera) Larval

Hemolymph in Response to

Autographa californica

Nucleopolyhedrovirus in

Differentially Resistant Strains.

Processes 2021, 9, 1401. https://

doi.org/10.3390/pr9081401

Academic Editors: Hoon Kim and Katarzyna Otulak-Kozieł

Received: 2 July 2021

Accepted: 6 August 2021

Published: 13 August 2021

Publisher's Note: MDPI stays neutral with regard to jurisdictional claims in published maps and institutional affiliations.

Copyright: (c) 2021 by the authors. Licensee MDPI, Basel, Switzerland. This article is an open access article distributed under the terms and conditions of the Creative Commons Attribution (CC BY) license (https:/ / creativecommons.org/licenses/by/ $4.0 /)$.

\begin{abstract}
Bombyx mori nucleopolyhedrovirus (BmNPV) is a kind of pathogen that causes huge economic losses to silkworm production. Although Autographa californica nucleopolyhedrovirus (AcMNPV) and BmNPV are both baculoviruses, the host domains of these two viruses have almost no intersection in nature. Recently, it has been found that some silkworms could be infected by recombinant AcMNPV through a puncture, which provided valuable material for studying the infection mechanism of baculovirus to silkworm. In this study, comparative transcriptomics was used to analyse the hemolymph of two differentially resistant strains following AcMNPV inoculation. There were 678 DEGs in p50 and 515 DEGs in C108 following viral infection. Among them, the upregulation and downregulation of DEGs were similar in p50; however, the upregulated DEGs were nearly twice as numerous as the downregulated DEGs in C108. The DEGs in different resistant strains differed by GO enrichment. Based on KEGG enrichment, DEGs were mainly enriched in metabolic pathways in p50 and the apoptosis pathway in C108. Moreover, 13 genes involved in metabolic pathways and 11 genes involved in the apoptosis pathway were analysed. Among the DEGs involved in apoptosis, the function of BmTex261 in viral infection was analysed. The BmTex261 showed the highest expression in hemolymph and a significant response to viral infection in the hemolymph of C108, indicating that it is involved in anti-AcMNPV infection. This was further validated by the significantly decreased expression of viral gene lef 3 after overexpression of BmTex261 in $\mathrm{BmN}$ cells. The results provide a theoretical reference for the molecular mechanism of resistance to BmNPV in silkworms.
\end{abstract}

Keywords: Bombyx mori; AcMNPV; transcriptome analysis; apoptosis; testis expressed genes 261

\section{Introduction}

Silkworm, Bombyx mori (B. mori), is one of the most important insects in developing countries because of the silkworm cocoon, which is used in the medical and food industries [1-4]. However, silkworm infection by pathogens is a common problem. For example, BmNPV causes serious losses, and the underlying defence mechanism is still unclear. AcMNPV is another representative baculovirus in insects and similar to BmNPV, except in the embedded ways [5]. Normally, both of them have strict host domains and generally do not cross-infect other host domains, but studies have found that AcMNPV can infect silkworms by puncturing [6]. This study further discussed the mechanism of the 
silkworm in response to baculovirus infection. The results in this study will be beneficial for clarifying the silkworm anti-BmNPV mechanism.

The baculoviruses are a family of large rod-shaped viruses that contain a circular, double-stranded genome ranging from 80 to $180 \mathrm{~kb}$. Baculoviruses are endemic to invertebrates, especially insects of the order Lepidoptera [7]. The BmNPV belongs to the Baculoviridae family and has closed circular double-stranded DNA genomes; it can only infect Bombycidae larvae [8]. The AcMNPV is one of the pathogens of Spodoptera frugiperda (S. frugiperda). Although it infects nearly 30 lepidopteron species, it has a narrow host range [7]. Both BmNPV and AcMNPV are highly similar. There are two distinct forms in the viral replication cycle, bud virus (BV) and occlusion-derived virus (ODV), with a similar nucleocapsid structure but differences in source, morphology, protein and capsule composition, target tissue specificity, and invasion mode. The BV is mainly produced during the early replication of baculoviruses [9-11]. BmNPV genome was over $90 \%$ identical to about three-quarters of the genome of AcMNPV. The relatedness of predicted amino acid sequences of corresponding ORFs between BmNPV and AcMNPV was about 90\% [12,13]; the largest difference between the two can be observed in the embedding methods. The BmNPV is a single-grain-embedded type, whereas AcMNPV is multi-grain embedded. Despite the high homology of the two genomic sequences, the host domains of the two virus types have almost no intersection in nature. For example, BmNPV can infect B. mori but not $S$. frugiperda, and vice versa. However, the recombinant AcMNPV can infect some silkworm strains by puncture, which is a peculiar phenomenon in the host domain of the virus [6].

Different strains of silkworms have different resistances to BmNPV in nature [14]. In recent years, researchers have studied the molecular mechanism of silkworm resistance to BmNPV from various aspects. For example, Xue et al. used the second-generation sequencing technology to analyse the differences in gene expression of Bm5 cell lines infected with BmNPV [15]. Gene enrichment analysis showed that the expression of genes related to cytoskeleton, transcription, translation, energy metabolism, ion metabolism, and the ubiquitin-proteasome pathway changed during baculovirus infection [15]. In addition, there have been some advances in proteomics [16-18]. For example, Hsp70 protein cognate, lipase-1, and chlorophyllide A-binding protein precursor were upregulated significantly after BmNPV challenge [19]. Arginine kinase was also found to be involved in the antiviral process of B. mori larvae against NPV infection at the protein level [20]. Other scholars have conducted a series of studies on lncRNA in silkworm resistance to BmNPV $[16,17]$. lncRNAs play a role in the regulation of BmNPV proliferation by Hsp90 [16]. DElncRNAs participated in the host response to BmNPV infection via interactions with their target genes and miRNAs [17]. However, the molecular mechanism of silkworm resistance to BmNPV infection has not been clearly studied.

Several studies have focused on the infection mechanism of AcMNPV in different insects. The recombinant virus of AcMNPV could be observed in midgut columnar cells of the second instar larvae of Spodoptera exigua at $3 \mathrm{~h}$ after oral inoculation and $6 \mathrm{~h}$ in regenerated cells [21]. The earliest expression time of the late viral gene in the columnar cells was $12 \mathrm{~h}$ after inoculation, which marked the replication of the virus [21]. Washburn et al. found that the early priming of GP64 reduced the oral infection of AcMNPV [22]. In addition to intracellular interactions, baculoviruses also encode gene products that function at the cellular level and thus manipulate the physiology and structure of infected animals. The gene product ecdysis hormone UDP-glycotransferase (EGT) prolongs the larval stage by inactivating the host's molting hormone, a steroid that regulates ecdysis. Viruses with this gene deleted can effectively shorten the lethal time, making them efficient pesticides [23]. The defect of AcMNPV as a Bac-to-Bac baculovirus expression system may cause cell death $[24,25]$. In addition, the dynamic process of nucleoactin aggregation, induced by baculovirus $\mathrm{p} 78 / 83$ protein to propagate progeny virus, has been reported [26-28]. Studies have confirmed that the nucleocapsid protein C42 of AcMNPV recruits P78/83 and ArP2/3 to mediate the aggregation of intranuclear actin $[26,27,29]$. By studying the molecular 
mechanism of the infection of AcMNPV-infected silkworms, we can prevent cell death caused by AcMNPV, obtaining high foreign protein yields [30].

Apoptosis is a common way of fighting off viruses [31,32]. As an "evolutionary response" to host cell apoptosis, baculoviruses carry genes that encode inhibitors of apoptosis [32]. Viral proteins can also inhibit apoptosis by inactivating proteins that bind to the apoptosis pathway [31]. Apoptosis induced by insect cells infected with baculovirus first occurs in the absence of the functional $p 35$ gene, which was observed in AcMNPV-infected insect S21 cells [33]. The $p 35$ is a direct substrate inhibitor of caspases [34]. Testis expressed gene 261 (Tex261) has been identified as a testis-specific protein in mice [35], but it is different in silkworms. Hideo Taniura and others found that Tex261 modulates the excitotoxic cell death induced by NMDA receptor activation [36], indicating that BmTex261 might be involved in cell apoptosis processes.

\section{Materials and Methods}

\subsection{Silkworm, AcMNPV, and Sample Preparation}

Silkworm strain p50 (susceptible strain) and C108 (resistant strain) were maintained in the Key Laboratory of Sericulture, School of Life Sciences, Jiangsu University of Science and Technology. The larvae were fed with fresh mulberry leaves in conditions of $26 \pm 1{ }^{\circ} \mathrm{C}$, $75 \pm 5 \%$ relative humidity, and a $12 \mathrm{~h}$ day /night cycle. The temperature was decreased to $24 \pm 1{ }^{\circ} \mathrm{C}$ in the last two instars, whereas the other conditions were not changed.

A budded virus of AcMNPV with an enhanced green fluorescent protein tag (BVEgfp) is maintained in the Key Laboratory of Sericulture, School of Life Sciences, Jiangsu University of Science and Technology. The titer of BV-eGFP was determined as described in LT et al. [37].

Silkworm larvae were injected with $2.0 \mu \mathrm{L}$ culture medium containing BV-eGFP $\left(1.0 \times 10^{8} \mathrm{pfu} / \mathrm{mL}\right)$ per larva on the first day of the fifth instar. The control group was injected with an equal volume of culture medium. The different tissues, including midgut, hemolymph, fat body, and malpighian tubule, were collected at $36 \mathrm{~h}$ after injection. The samples were frozen in liquid nitrogen, immediately powdered, and stored at $-80{ }^{\circ} \mathrm{C}$ until use.

\subsection{Library Construction, Illumina Sequencing, and Read Assembly}

Transcriptome analysis was performed by Beijing Novogene Technology (Beijing, China), mainly including RNA extraction, library preparation, clustering, sequencing, and assembling. A total of $1.0 \mu \mathrm{g}$ RNA was used for library construction. The libraries were constructed applying the NEB Next ${ }^{\circledR}$ Ultra $^{\mathrm{TM}}$ RNA Library Prep Kit for Illumina ${ }^{\circledR}$ (NEB, Ipswich, Massachusetts, USA). The mRNA was purified from total RNA using poly-T oligomagnetic beads. The first chain synthesis reaction buffer $(5 \times)$ was cleaved using divalent cations at high temperatures. The first-strand cDNA was synthesised using random hexamer primers and M-MuLV Reverse Transcriptase (RNase H-). Subsequently, the second-strand cDNA was synthesised using DNA Polymerase I and RNase H. The remaining overhangs were converted to blunt ends by exonuclease/polymerase activities. After the $3^{\prime}$ ends of the DNA fragment were adenylated, NEB Next Adaptor with hairpin ring structure was linked to prepare for hybridisation. To select cDNA fragments of preferentially $250-300 \mathrm{bp}$ in length, the library fragments were purified by the AMPure $\mathrm{XP}$ system. Then, $3 \mu \mathrm{L}$ of USER Enzyme (US NEB) was used to the select size and connect the adapters to cDNA at $37{ }^{\circ} \mathrm{C}$ for $15 \mathrm{~min}$, with $95^{\circ} \mathrm{C}$ for $5 \mathrm{~min}$ before PCR. Phusion High-Fidelity DNA polymerase, Universal PCR primer and Index (X) primer were used for PCR; the PCR products were purified (AMPure XP system), and library quality was evaluated by the Agilent Bioanalyzer 2100 system. The raw reads were cleaned by Fastp and evaluated by the FastQC programme [38]. Hisat2 was used to map reads to reference genome from SilkDB3.0 [39-42]. Stringtie was used to assemble the novel transcript based on the mapping results [43]. FeatureCounts was used to obtain raw count of every gene. All the programmes were used with default parameters. 


\subsection{Functional Annotation and Enrichment Analysis}

To annotate unigenes which were assembled by Stringtie, sequences were searched by BLASTx against the NCBI non-redundant protein (nr) database and other databases, including the Swiss-Prot protein database, the Kyoto Encyclopedia of Genes and Genomes (KEGG) and the Cluster of Orthologous Groups (COG) databases. The R package clusterProfile was used to perform KEGG and GO enrichment [30,31]. EuKaryotic Orthologous Groups (KOG) is a eukaryote-specific version of the Clusters of Orthologous Groups (COG) tool for identifying orthologue and paralogue proteins. The KOG classification of DEGs was performed by blast against KOG database of NCBI [44,45]. All searches were performed with an E-value $<10^{-5}$. The other genes have been fully annotated in SilkDB3.0.

\subsection{Identification of Differentially Expressed Genes (DEGs)}

The fragments per kilobase of transcript per million fragments mapped (FPKM) were calculated to represent the expression abundance of the unigenes [32]. Feature Counts were used to count the read numbers mapped to each gene, and the FPKM of each gene was calculated based on the length of the gene and the read count mapped to this gene. The FPKM, the expected number of Fragments Per Kilobase of transcript sequence per million base pairs sequenced, simultaneously considers the effects of sequencing depth and gene length for the read count and is currently the commonly used method for estimating gene expression levels. FPKM was calculate based on the formula and calculated in R:

$$
F P K M=\frac{\text { ExonMappedReads } * 10^{9}}{\text { TotalMappedReads } * \text { Exonlength }}
$$

The DESeq2 R package (https:/ / genomebiology.biomedcentral.com/articles/10.118 6/s13059-014-0550-8, accessed on November 2020) was used to analyse the differential expression between two groups; it uses a model based on the negative binomial distribution to provide statistical routines for determining differential expressions in digital gene expression data. The resulting $p$-values were adjusted by using Benjamini and Hochberg's approach to control the false discovery rate (FDR $<0.03$ ). Genes with an adjusted $p$-value $<0.05$ by DESeq 2 were assigned as differentially expressed. Genes with Log2FoldFC $\geq 1$ were selected.

\subsection{RNA Extraction, First-Strand cDNA Synthesis, and Real-Time Quantitative PCR (RT-qPCR)}

The total RNA was extracted using RNAiso Plus according to the manufacturer's instructions, purified by the isopropanol precipitation and dissolved in DEPC water. The assessing optical density (OD) absorbance ratio of $260 / 280$ was used to determine RNA purity. The concentration of RNA was detected using a NanoDrop 2000 spectrophotometer. The integrity of RNA was checked by $1 \%$ agarose gel electrophoresis. A total of $1.0 \mu \mathrm{g}$ of RNA was reverse-transcribed in vitro by the PrimeScript ${ }^{\mathrm{TM}} \mathrm{RT}$ reagent kit according to the manufacturer's instructions.

RT-qPCR was used to detect the gene expression levels. The specific primers used in RT-qPCR were designed by the NCBI Primer-BLAST software (https:/ / www.ncbi.nlm. nih.gov/tools/primer-blast/, accessed on January 2021) and are shown in Table 1. The reaction mixtures were prepared using the NovoStart ${ }^{\circledR}$ SYBR qPCR SuperMix Plus kit (Novoprotein Technology Ltd., China) according to the manufacturer's instructions. Briefly, a $15 \mu \mathrm{L}$ qPCR reaction system was used, including $7.5 \mu \mathrm{L}$ of $2 \times$ NovoStart $^{\circledR}$ SYBR qPCR SuperMix Plus, $0.5 \mu \mathrm{L}$ of upstream and downstream primers, $1.5 \mu \mathrm{L}$ of the template, and $5.0 \mu \mathrm{L}$ of $\mathrm{dd} \mathrm{H}_{2} \mathrm{O}$. The reactions were performed on the LightCycler ${ }^{\circledR} 96$ System (Roche, Basel, Switzerland). The following qPCR protocol was used: one cycle at $95^{\circ} \mathrm{C}$ for $5 \mathrm{~min}$, followed by 40 cycles at $95^{\circ} \mathrm{C}$ for $20 \mathrm{~s}$, and $60^{\circ} \mathrm{C}$ for $60 \mathrm{~s}$. The $2^{-\Delta \Delta C \mathrm{~T}}$ method was adopted to calculate the relative expression level. Each group was repeated three times. B. mori glyceraldehyde-3-phosphate dehydrogenase $(B m G A P D H)$ was used as the reference gene. The late expression factor 3 (lef3) was used to detect AcMNPV (Table 1). 
Table 1. List of primers used in this study.

\begin{tabular}{|c|c|c|c|}
\hline No. & Primer Names & Forward Primer $\left(5^{\prime}-3^{\prime}\right)$ & Reverse Prime $\left(5^{\prime}-3^{\prime}\right)$ \\
\hline 1 & BMSK0012268 & CAGGCGATGAAGCTGGAGAA & GCGGACTTCCTCGTTTACCT \\
\hline 2 & BMSK0012667 & GGCGAAGCAAAATGGCAGAA & ATTTGACGCGCTTATCGTGC \\
\hline 3 & BMSK0013103 & CCAACTCAGCTAGACGATGCC & GATGCCAAGTTCCCGAAGATAG \\
\hline 4 & BMSK0005642 & AACTCTGGCCGCTAAGTTCA & TCAGCTGCTCGTCCAATTCC \\
\hline 5 & BMSK0015907 & AAAGACCAACGGAACTGCGA & CCTGTGAATTCGGTCCCCTC \\
\hline 6 & BMSK0013101 & ACCGCACGGGAACTAGGA & CCAAGCCTAGATGCTCGTTGT \\
\hline 7 & BMSK0000440 & GCAGTTCCGGTGAAGAGACA & AAGAAGGAGGTGGGAAGGGA \\
\hline 8 & BMSK0008569 & AAAACACGCCCGATTCACAC & CGCGACTGTAAGTGGGAGAA \\
\hline 9 & BMSK0009445 & TGCTACAGACGAGACTACCC & TGGATCTGTTCGCCCCTT \\
\hline 10 & BMSK0014619 & CCGACATTGTTTGCCGTTGT & GCACTTCTGGTTGATGATGCC \\
\hline 11 & BMSK0000576 & TAAACAAGGTCGGTCACGCA & GCCGTTTTGAACTGTGGCTT \\
\hline 12 & BmTex261 & CGTGTTGCCAACGACAGAAG & CGCTTTCTTGTTCCGGTGAG \\
\hline 13 & $B m G A P D H$ & CCGCGTCCCTGTTGCTAAT & CTGCCTCCTTGACCTTTTGC \\
\hline 14 & lef3 & CAAACGCGTTGCTTCGTACA & TGCTCGAGTCGGAAGAGGTA \\
\hline 15 & BmTex261 KX & GGGGTACCATGTTATTCTTGTATTTATTGAGTTATT & GCTCTAGAGAACGCTTTCTTGTTCCG \\
\hline
\end{tabular}

Notes: No. 1-11 are primers for RT-qPCR validation of differentially expressed transcript; No. 12-14 are primers to detect gene expression by RT-qPCR; No. 15 is the primer to amplify the CDS of BmTex261.

\subsection{Construction of pIZT-mCherry-BmTex261 Overexpression Vector}

The primers that amplify the coding sequence of BmTex261 were designed with reference to NCBI-designed primers (Table 1; underlined bases are the restriction sites). The sequence was amplified from cDNA of BmN cell, and the purified products were cloned with the pMD-19T vector and sequenced by SUNYA Biotechnology (Zhejiang, China). The sequence with the construct without mutations was inserted into the pIZT/V5His-mCherry expression vector with T4 DNA ligase (TaKaRa Biotechnology Co. Ltd., Kyoto, Japan) and confirmed by enzyme cutting via Kpn I and Xba I (TaKaRa Biotechnology Co. Ltd., Kyoto, Japan).

\subsection{Synthesis of siRNA}

To knockdown BmTex261, two targets, located in the functional domain, were designed by Thermo Fisher Scientific BLOCK-iT ${ }^{\mathrm{TM}}$ RNAi Designer (https:/ / rnaidesigner. thermofisher.com/rnaiexpress/, accessed on May 2020). The siRNAs' oligos were synthesised by SUNYA Biotechnology (Zhejiang, China) and are listed in Table 2. The siRNAs were synthesised by the In Vitro Transcription T7 Kit (TaKaRa Biotechnology Co. Ltd., Japan) according to the manufacturer's instructions; siRNA, which was synthesised by BmTex261-1 Olig (siTex261), was used to knockdown the expression of BmTex261 in BmN cells, and the siRNA which was synthesised by RFP-Olig was used as the control. The kit uses the T7 RNA polymerase, which takes the linear double-stranded DNA containing the T7 promoter sequence as the template and can transcribe the DNA sequence downstream of the promoter to efficiently synthesise single-stranded RNA. The absorbance ratio of $260 / 280$ and the concentration were detected by a NanoDrop 2000 spectrophotometer (Thermo Scientific, New York, NY, USA). The quality of synthesised siRNAs was checked by $3 \%$ agarose gel electrophoresis at $160 \mathrm{~V}$ for $8 \mathrm{~min}$. The qualified siRNAs were stored at $-80{ }^{\circ} \mathrm{C}$ until use. 
Table 2. List of primer sequences used to synthesise siRNA.

\begin{tabular}{cc}
\hline Primer Names & Sequences $\left.\mathbf{( 5}^{\prime} \mathbf{s}^{\prime} \mathbf{}^{\prime}\right)$ \\
\hline BmTex261-1 Olig-1 & GATCACTAATACGACTCACTATAGGGAAGTCATCACGTATGCTGTATTT \\
BmTex261-1 Olig-2 & AAATACAGCATACGTGATGACTTCCCTATAGTGAGTCGTATTAGTGATC \\
BmTex261-1 Olig-3 & AAAAGTCATCACGTATGCTGTATCCCTATAGTGAGTCGTATTAGTGATC \\
BmTex261-1 Olig-4 & GATCACTAATACGACTCACTATAGGGATACAGCATACGTGATGACTTTT \\
BmTex261-2 Olig-1 & GATCACTAATACGACTCACTATAGGGAACGTTCTGACGGATTATCTGTT \\
BmTex261-2 Olig-2 & AACAGATAATCCGTCAGAACGTTCCCTATAGTGAGTCGTATTAGTGATC \\
BmTex261-2 Olig-3 & AAAACGTTCTGACGGATTATCTGCCCTATAGTGAGTCGTATTAGTGATC \\
BmTex261-2 Olig-4 & GATCACTAATACGACTCACTATAGGGCAGATAATCCGTCAGAACGTTTT \\
RFP-Olig-1 & GATCACTAATACGACTCACTATAGGGGCACCCAGACCATGAGAATTT \\
RFP-Olig-2 & AAATTCTCATGGTCTGGGTGCCCCTATAGTGAGTCGTATTAGTGATC \\
RFP-Olig-3 & AAGCACCCAGACCATGAGAATCCCTATAGTGAGTCGTATTAGTGATC \\
RFP-Olig-4 & GATCACTAATACGACTCACTATAGGGATTCTCATGGTCTGGGTGCTT \\
\hline
\end{tabular}

\subsection{BmN Cell Culture and Transfection}

The BmN cell line was obtained from silkworm ovary. It was cultured in TC100 medium pH 6.2 with 10\% fetal bovine serum (FBS) and 1\% penicillin-streptomycin solution at $28{ }^{\circ} \mathrm{C}$. The culture medium was replaced every 4 days.

The overexpression plasmid and dsRNA were transfected by Neofect ${ }^{\mathrm{TM}}$ DNA transfection reagent according to the manufacturer's instructions. The BmN cells were cultured in the dish. Briefly, each $60 \mathrm{~mm}$ dish required $4.0 \mu \mathrm{g}$ plasmid or dsRNA. The plasmid and dsRNA were mixed with $200 \mu \mathrm{L}$ TC100 without FBS and $4.0 \mu \mathrm{L}$ Neofect transfection reagent. The highest transfection efficiency was determined for different durations.

\subsection{Statistical Analysis}

The statistical differences among three biological duplicates were determined with ANOVA and Tukey's tests, using graphpad prism. The level of statistical significance was set at ${ }^{*}, p<0.05,{ }^{* *}, p<0.01$, and ${ }^{* * *}, p<0.001$.

\section{Results}

\subsection{Analysis of AcMNPV Infection in Differentially Resistant Strains}

In previous studies, we found that the recombinant AcMNPV-eGFP could be detected in p50 by puncture but not the C108 strain [37]. The epidermis of p50 infected with AcMNPV gradually changed from white to transparent, and the p50 could still spin silk but had abnormal cocooning and could not metamorphose into pupae (Figure 1A). All silkworms (100\%) infected with AcMNPV showed this phenomenon (Figure 1A), in contrast to the p50 infected with BmNPV. The other three groups were consistent with normal silkworms (Figure 1B-D). After p50 was infected with BmNPV, the body swelled up and the epidermis ruptured, resulting in the death of p50 and the exudation of white pus. Moreover, infection with AcMNPV in silkworm individuals was also determined at different durations in a previous study; the peak of AcMNNPV proliferation occurred at $36 \mathrm{~h}$ after inoculation with BV-eGFP $\left(1.0 \times 10^{8} \mathrm{pfu} / \mathrm{mL}\right)$ in p50 strains [37], and this time point was selected for further sample preparation. 


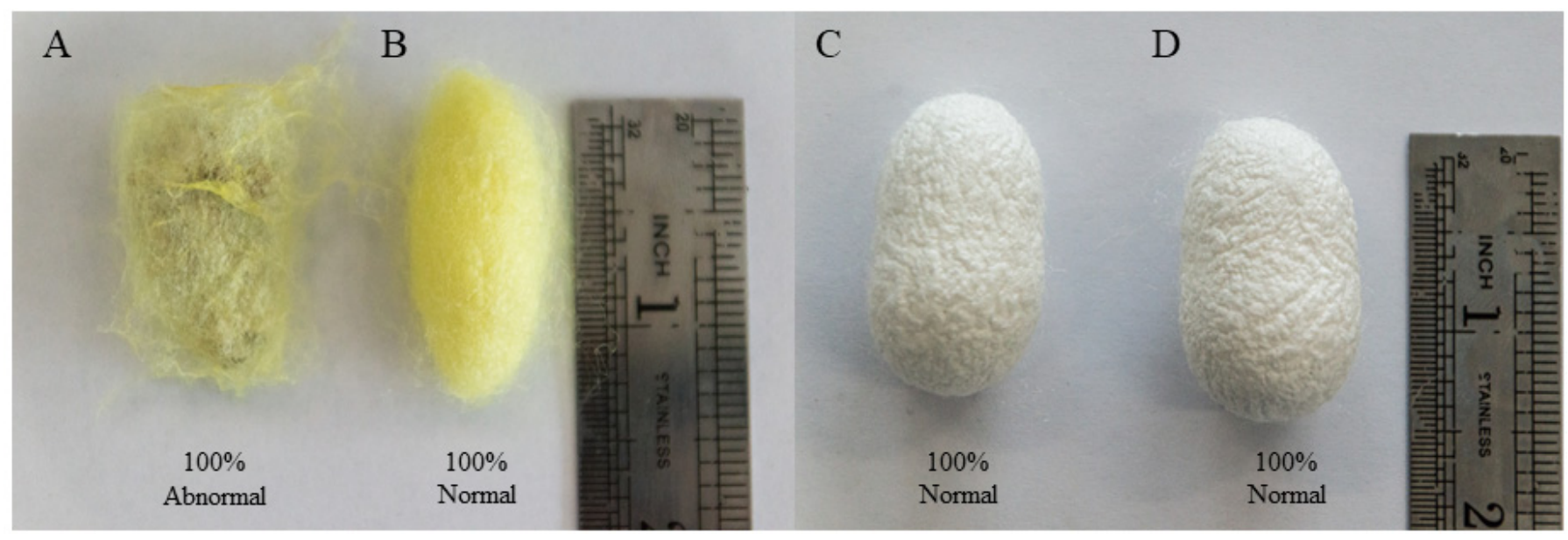

Figure 1. Phenotype of different resistant strains after AcMNPV inoculation. (A) p50 was infected with AcMNPV, (B) p50 without treatment, (C) C108 was infected with AcMNPV, and (D) C108 without treatment.

\subsection{Overview of the Transcriptome of Silkworm Hemolymph}

To systematically investigate the molecular mechanism of silkworm response against AcMNPV infection, transcriptome analysis was performed on the hemolymph of differentially resistant silkworm strains following AcMNPV inoculation at $36 \mathrm{~h}$. Four cDNA libraries were constructed, including p50 - (control group), p50+ (inoculated with AcMNPV), C108- (control group), and C108+ (inoculated with AcMNPV). The GC content of the four libraries was about $50 \%$, and all Q30\% were equal to or greater than $93.27 \%$ (Table 3). A total of 181,323,874 reads were obtained by analysing high-quality sequences (Table 3). The results indicated that the sequencing data were qualified and could be further analysed. Principal component analysis (PCA) analysis showed that the sequencing data of p50 - were significantly different from the other two, and subsequent analyses excluded this group (Supplementary Figure S1). To keep the remaining two sets of data available, the data were re-analysed again by the bio-company, and the results showed the data could be used for further analysis.

Table 3. Summary statistics for silkworm genes based on RNA-seq data.

\begin{tabular}{ccccc}
\hline & p50- & p50+ & C108- & C108+ \\
\hline Total Reads & $44,728,956$ & $46,036,772$ & $46,693,816$ & $43,864,330$ \\
GC Content (\%) & 48 & 46 & 48 & 48 \\
\% $\geq$ Q30 (\%) & 93.35 & 93.36 & 93.27 & 94.49 \\
Mapped Reads & $41,118,461$ & $33,412,593$ & $39,765,815$ & $42,254,696$ \\
Mapped Ratio (\%) & 91.92 & 72.72 & 90.66 & 90.46 \\
Unique Mapped Reads & $34,225,042$ & $30,271,861$ & $37,902,000$ & $39,895,987$ \\
Unique Mapped Ratio (\%) & 85.00 & 65.87 & 86.41 & 85.44 \\
\hline
\end{tabular}

Note: Q30 means the percentage of bases with mass value $\geq 30$.

\subsection{RT-qPCR Validation of Differentially Expressed Transcripts}

To verify the reliability of transcriptome data, the relative expression levels of 11 randomly selected DEGs were analysed by RT-qPCR (Figure 2A-K). The trends of differential expression of these genes were consistent with the transcriptome data. Linear regression was performed for the correlation between RT-qPCR and RNA-seq data (Figure 2L); $\mathrm{R}^{2}$ was 0.896, with a slope of 1.18 (Figure 2L), indicating a positive correlation between RT-qPCR and transcriptome data. In summary, we consider the data of transcriptome to be credible. 
A

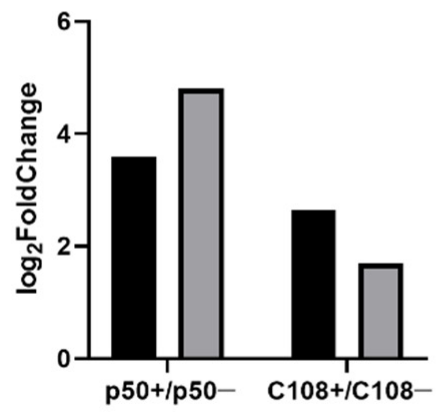

D
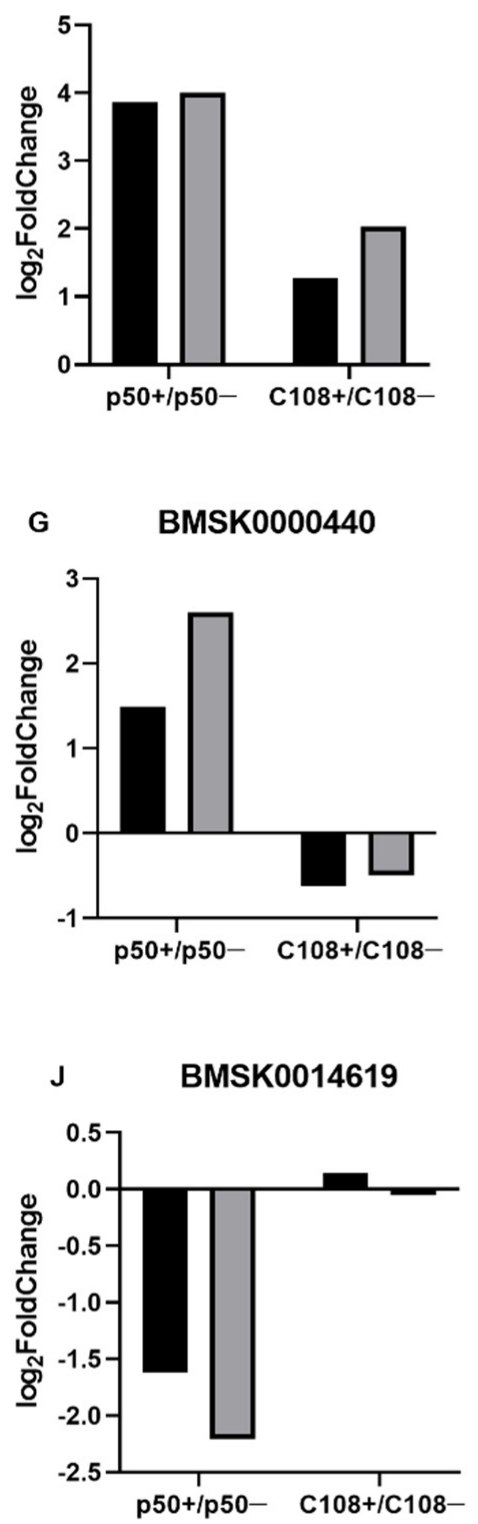

B

BMSK0012667

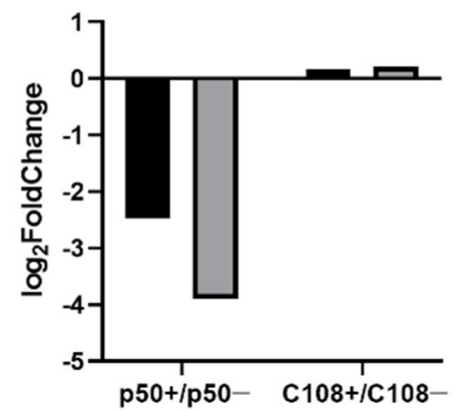

E

BMSK0015907

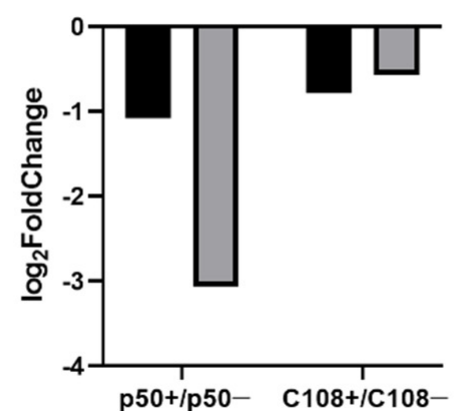

H $\quad$ BMSK0008569

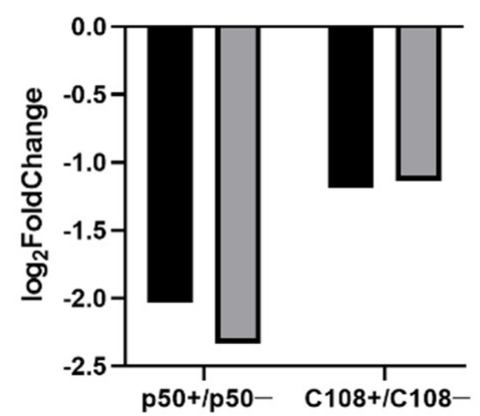

K

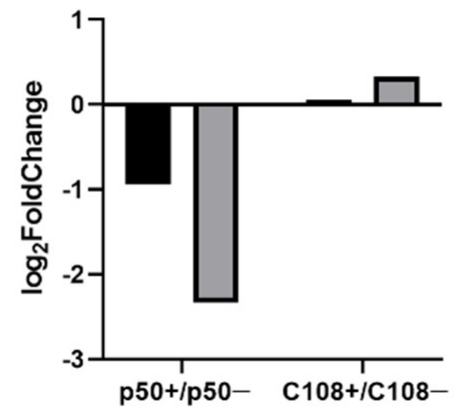

C BMSK0013103

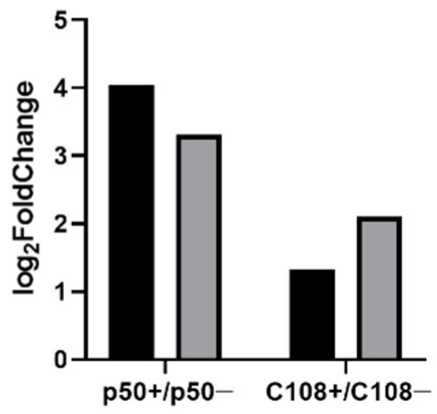

F

BMSK0013101
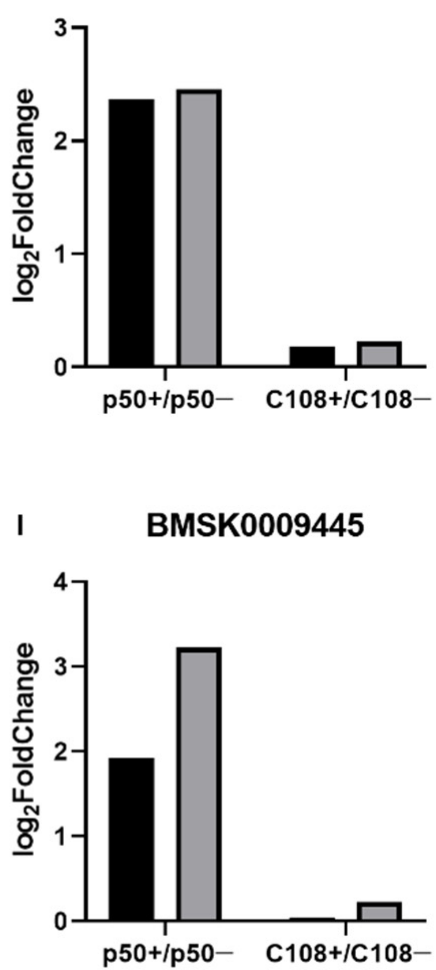

L

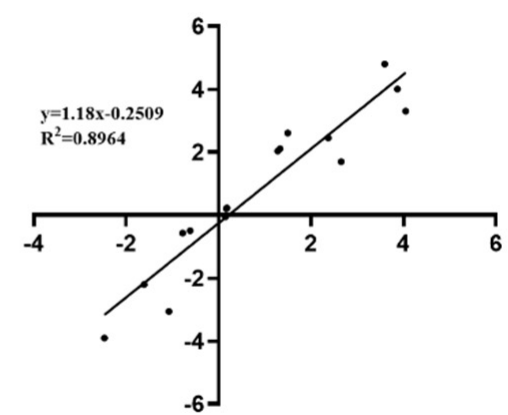

Figure 2. Correlation analysis between gene expression ratios obtained from transcriptome data and RT-qPCR. (A-K) Expression ratios ( $\log _{2}$ fold change) were obtained from RT-qPCR and transcriptome data. (L) Lineage analysis between the transcriptome and RT-qPCR. The ratios obtained by RT-qPCR ( $x$-axis) were plotted against the ratios obtained by RNA-Seq (y-axis). 


\subsection{Detection and Enrichment Analysis of DEGs}

The DEGs with significant differences were analysed from transcriptomic data when the difference was more than two times, and the adjusted $p$ value was less than 0.05 ; a total of 1402 genes were generated. In the p50+ vs. p50 - group, it resulted in 691 DEGs, including 391 upregulated and 313 downregulated DEGs (Table 4). In the C108+ vs. C108group, there were 515 DEGs, with 336 upregulated and 179 downregulated DEGs (Table 4). All DEGs have been listed in Supplementary Figures S1 and S2.

Table 4. Statistical analysis of DEGs in two strains following inoculation.

\begin{tabular}{ccccc}
\hline Groups & DEGs & Ratio of Total Transcripts & Upregulation & Downregulation \\
\hline p50+ vs. p50- & 679 & $6.73 \%$ & $301(44.33 \%)$ & $378(55.67 \%)$ \\
C108+ vs. C108- & 515 & $5.04 \%$ & $336(65.24 \%)$ & $179(34.76 \%)$ \\
\hline
\end{tabular}

The GO enrichment analysis showed that in silkworms with different resistances, DEGs were enriched in different categories (Figure 3). In the biological process, DEGs were mainly enriched in binding activity, but there were different binding types. Some cellular components, such as vesicle, endosome, and biological regulation in molecular function, exhibited opposite expression patterns of up- and downregulation in the different resistant strains; they were upregulated in p50+ vs. p50- and downregulated in C108+ vs. C108-.
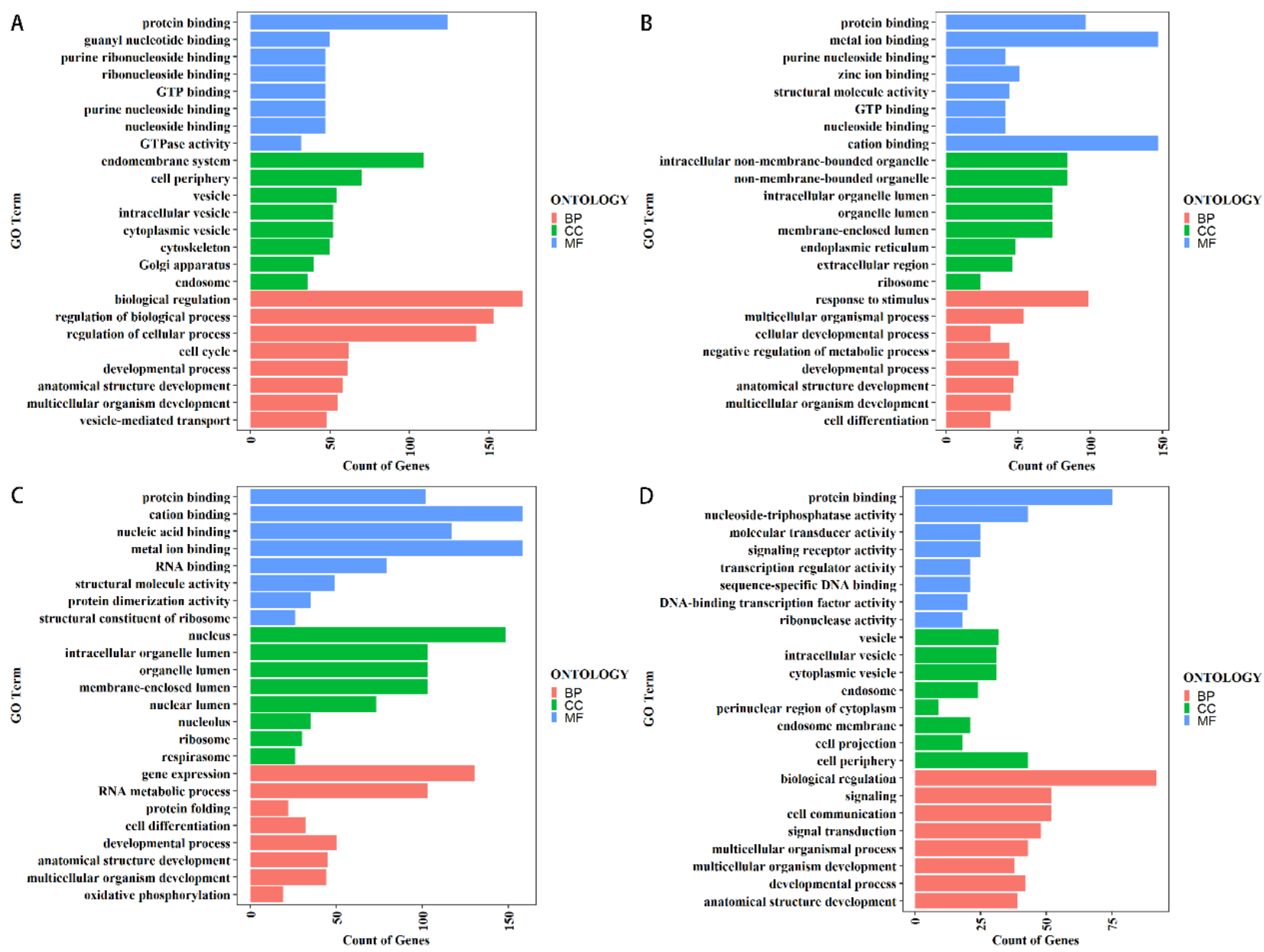

Figure 3. GO analysis of DEGs in differentially resistant strains following inoculation. These genes were divided into three groups based on cellular components. Biological process (BP), cellular component (CC), and molecular function (MF). (A) Upregulated DEGs in p50+ vs. p50- group, (B) upregulated DEGs in C108+ vs. C108- group, (C) downregulated DEGs in p50+ vs. p50 - group, (D) downregulated DEGs in C108+ vs. C108- group. 
The KEGG enrichment showed that DEGs mainly enriched in 16 metabolic pathways in p50+ vs. p50 - (Figure 4A,C). For example, sulfur metabolism and linoleic acid metabolism were the most two enriched terms in p50; 10 DEGs in C108+ vs. C108- were enriched in the apoptosis pathway (Figure 4D).

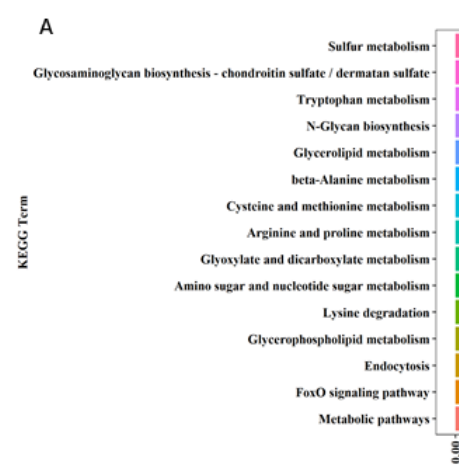

C

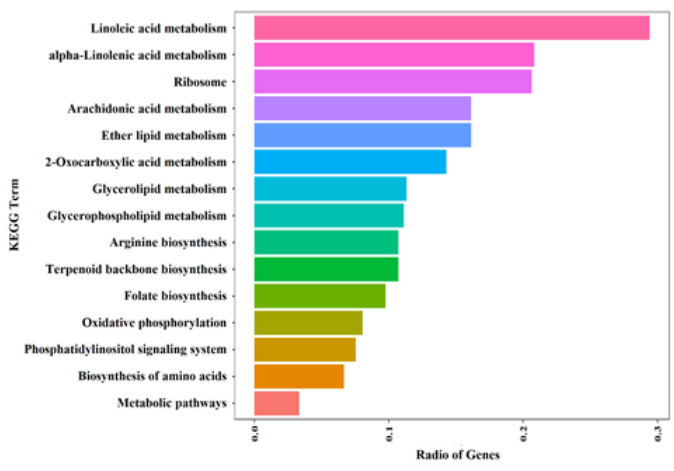

B
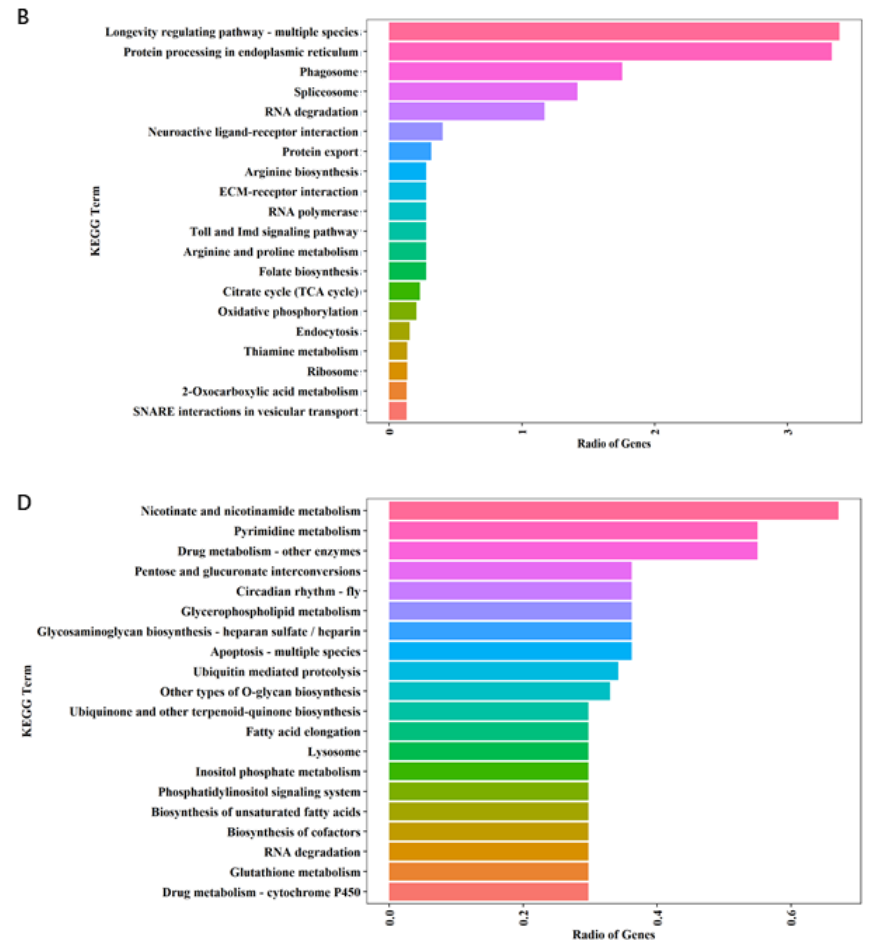

Figure 4. KEGG pathway enrichment analysis of DEGs in differentially resistant strains following inoculation. (A) Upregulated DEGs in p50+ vs. p50 - group, (B) upregulated DEGs in C108+ vs. C108- group, (C) downregulated DEGs in p50+ vs. p50- group, (D) downregulated DEGs in C108+ vs. C108- group.

The KOG annotation showed that six metabolism-related families in p50 were more than those in C108, especially in carbohydrate transport and metabolism, and inorganic ion transport and metabolism (Figure 5). In addition, DEGs in p50 of translation, ribosomal structure, and biogenesis were significantly more than that in C108 (Figure 5). The results were consistent with those in GO and KEGG. 
A
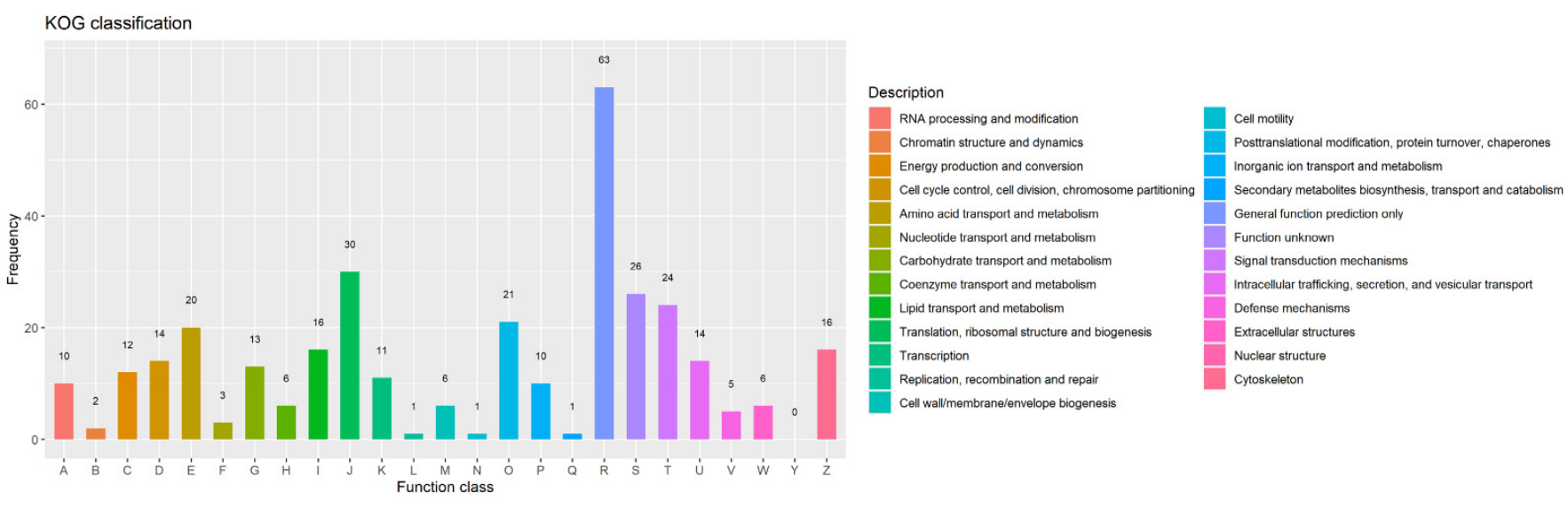

B
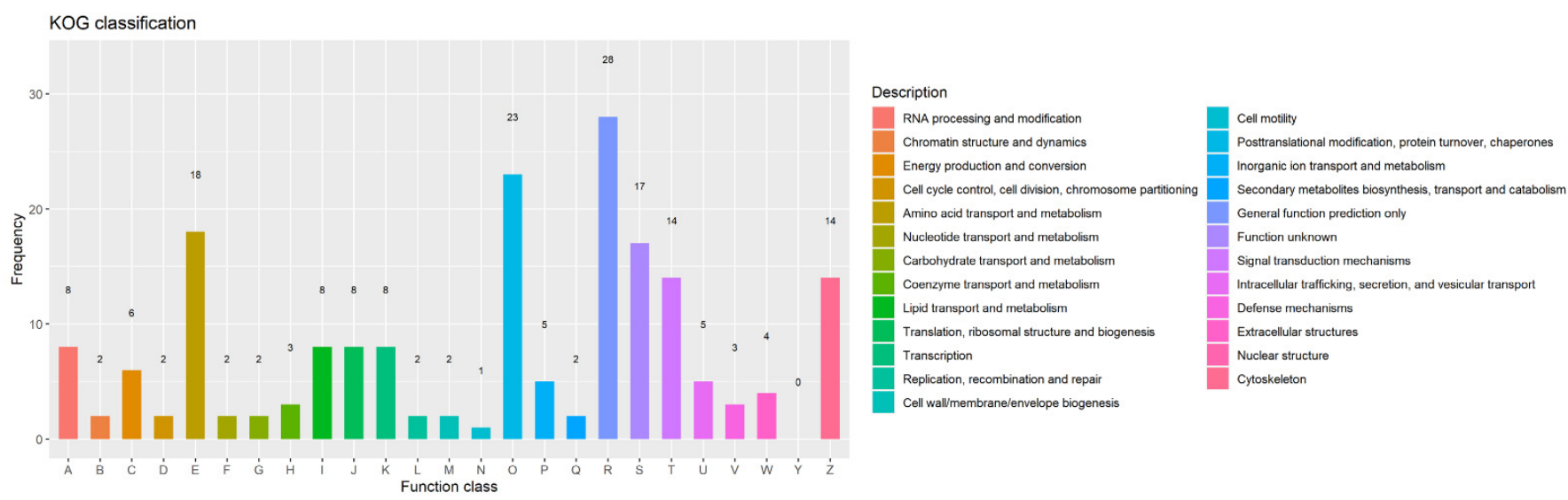

Figure 5. KOG annotation of DEGs in differentially resistant strains following inoculation. (A) DEGs in p50+ vs. p50group, (B) DEGs in C108+ vs. C108- group.

3.5. DEGs Involved in Metabolic and Apoptosis Pathways Showed Significant Responses to AcMNPV Infection

Based on the KEGG analysis, most DEGs in p50+ vs. p50- were enriched in the metabolic pathway. In addition, some DEGs were enriched in the apoptosis pathway. These two pathways are associated with viral infections, making it reasonable to speculate that the DEGs listed in the two pathways (Table 5) are related to the mechanism of silkworm defence to AcMNPV infection. 
Table 5. DEGs involved in metabolic pathways and apoptosis pathways.

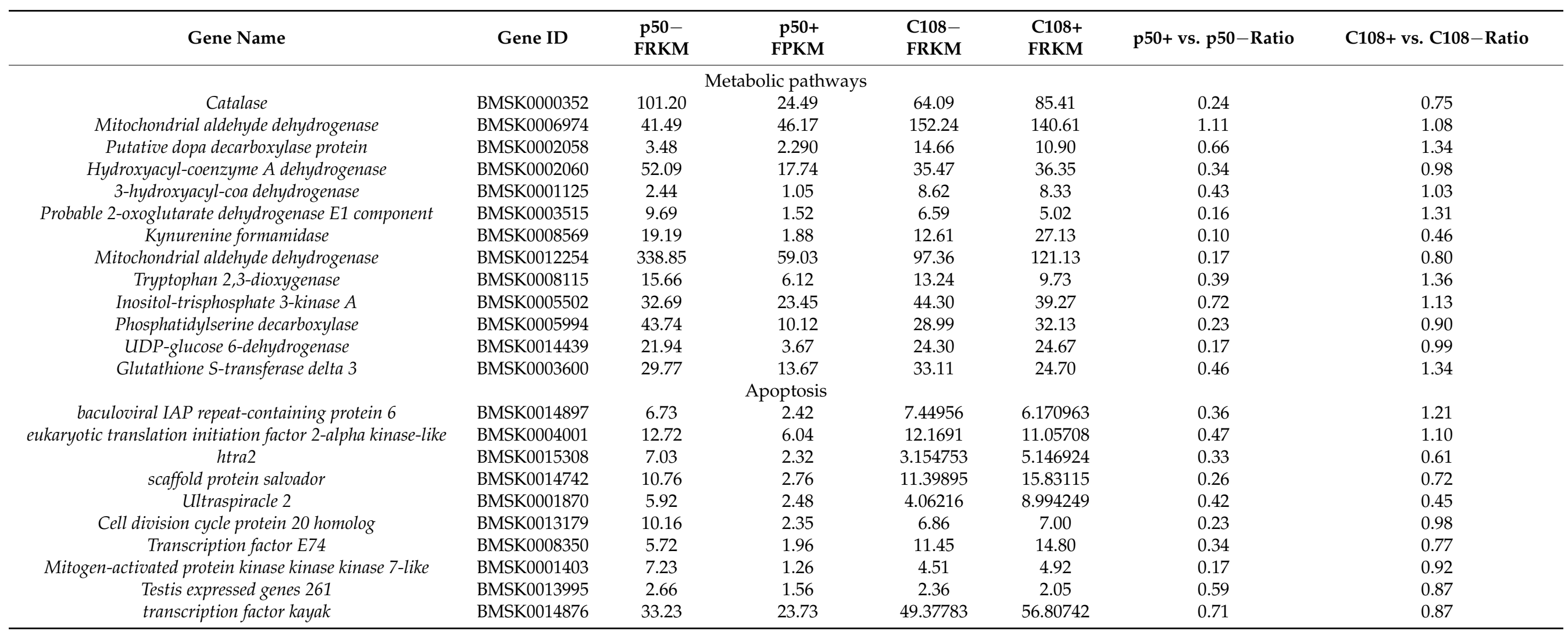




\subsection{Spatiotemporal Expression Pattern of B. mori Testis Expressed Genes 261 (BmTex261)}

To preliminarily study the biological function of BmTex261, the expression levels of BmTex261 in different tissues of fifth instar larvae and developmental stages in p50 silkworm strain were detected by RT-qPCR. The expression level of BmTex261 was highest in the hemolymph compared to other tissues. Moreover, BmTex261 was relatively low among other tissues, without significant differences among tissues (Figure 6). In different developmental stages, BmTex261 was expressed throughout all selected developmental stages, with the highest levels in the pupal stage.

A

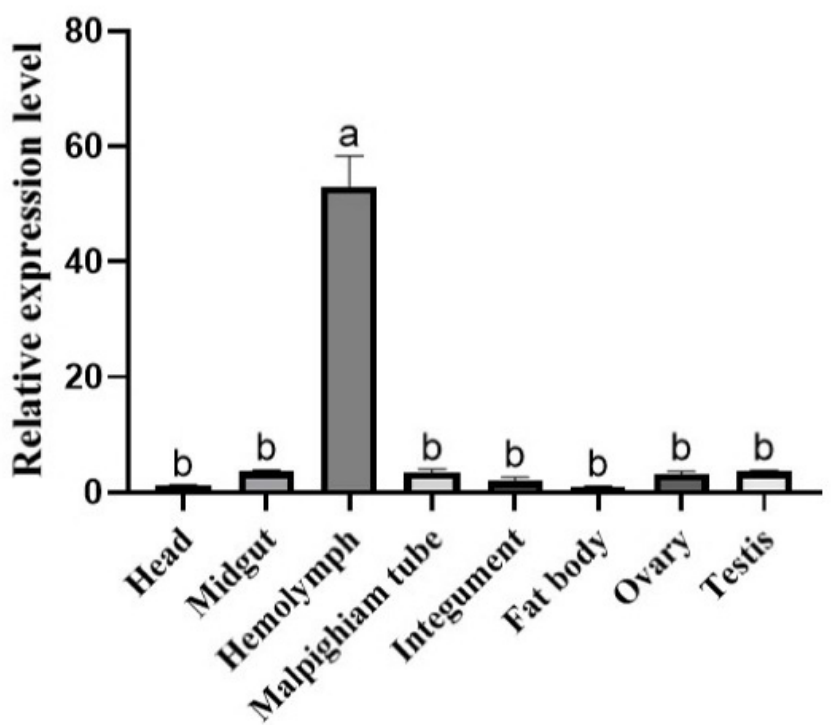

B

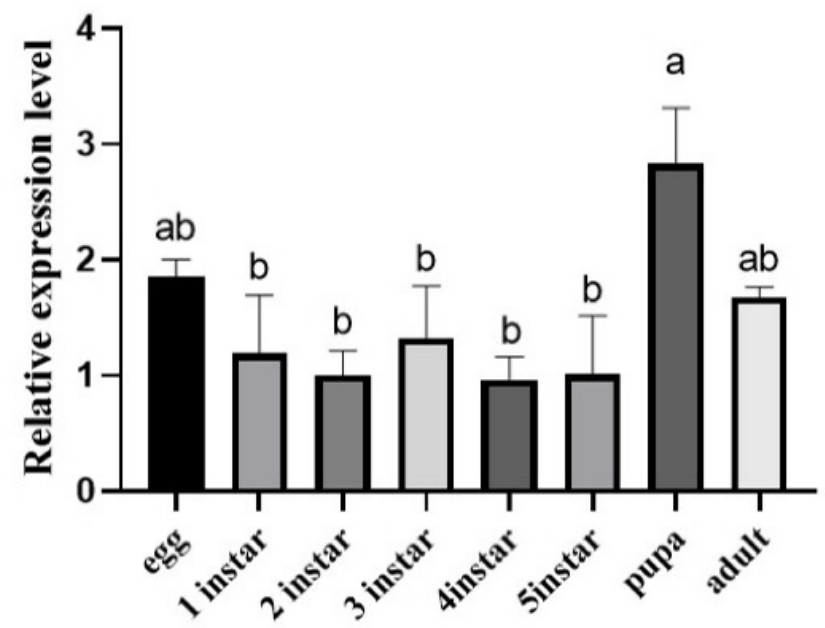

Figure 6. Expression analysis of BmTex261 in different tissues and developmental stages using RT-qPCR. (A) Relative expression levels of BmTex261 among different tissues; (B) relative expression levels of BmTex261 among different stages. $B m G A P D H$ was used to normalise the data that are shown as the mean \pm standard error; the mean was obtained from three independent repeats. The $2^{-\Delta \Delta \mathrm{Ct}}$ method was adopted to calculate the relative expression level. The statistical difference among three biological duplicates, determined with one-way ANOVA, is shown via graphpad prism. Different letters indicate statistically significant differences $(a, b ; p<0.05)$.

\subsection{Response Analysis of BmTex261 to AcMNPV Infection}

The expression levels of BmTex261 in the hemolymph, midgut, malpighian tubule, and fat body in p50 (susceptible strain) and C108 (resistant strain) were detected after injection with AcMNPV. The expression levels of BmTex261 in the hemolymph of the p50 strain were significantly downregulated after injection with AcMNPV as compared to the control group (Figure 7), whereas the expression of BmTex261 was upregulated in the midgut of p50 (Figure 7). 

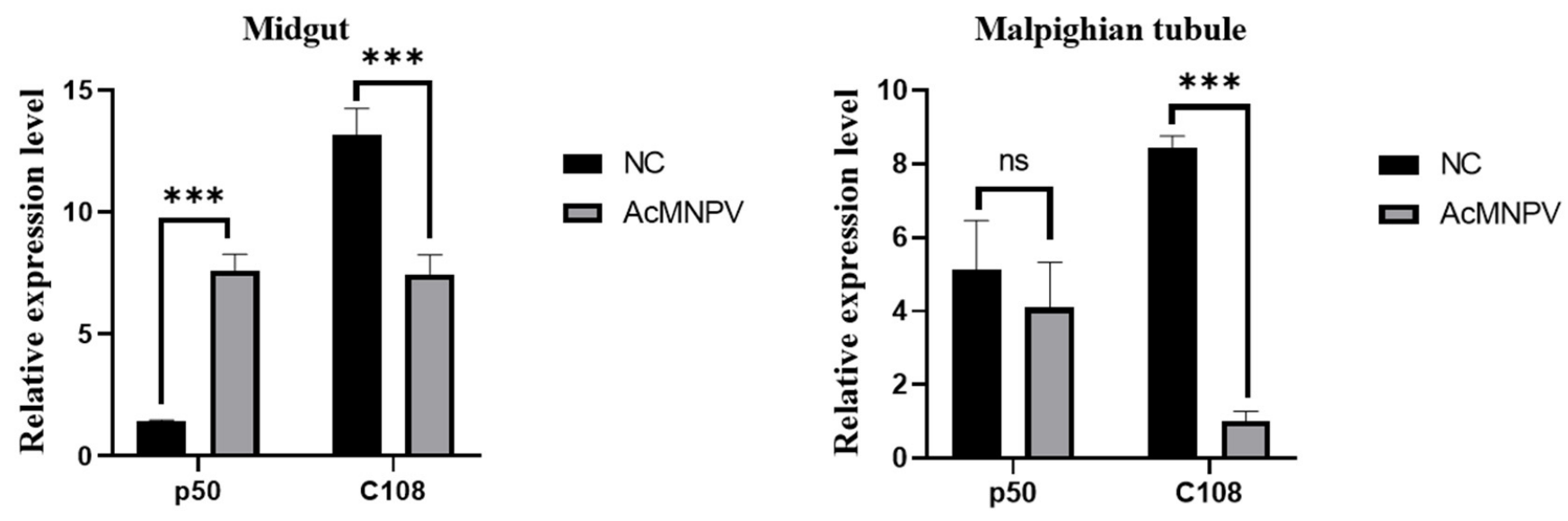

Fat body

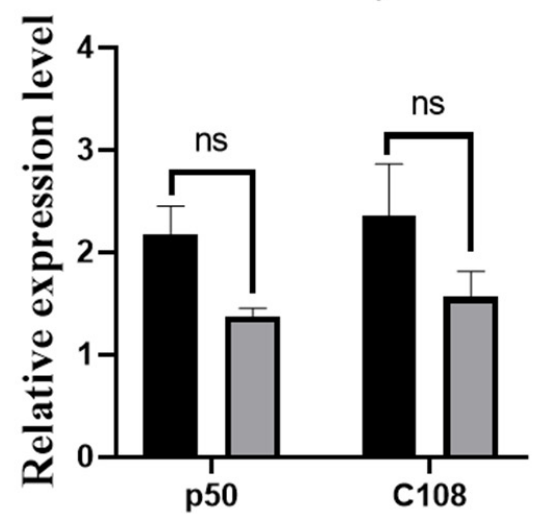

NC
AcMNPV

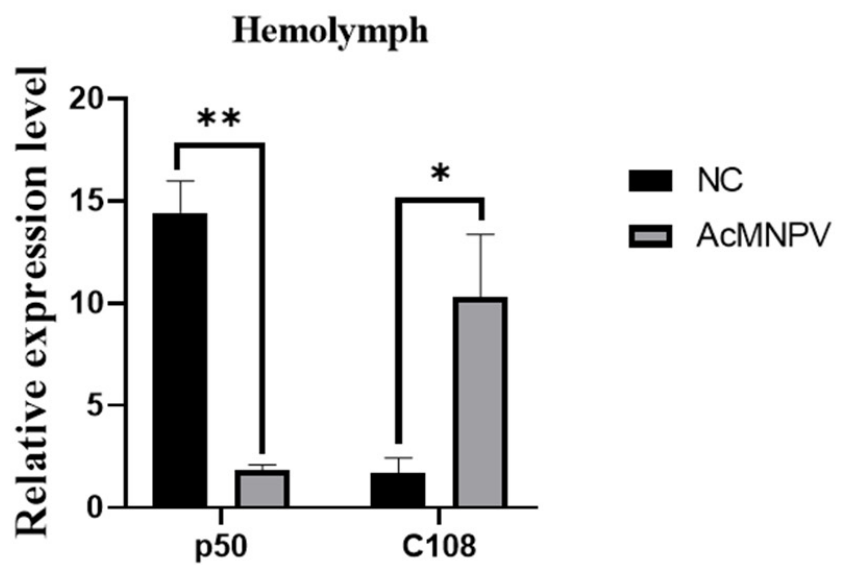

Figure 7. Analysis of BmTex261 expression levels in different tissues among different resistant strains following AcMNPV infection using RT-qPCR. NC is the negative control. BmGAPDH was used to normalise the data that are shown as the mean \pm standard error; the mean was obtained from three independent repeats. The $2^{-\Delta \Delta \mathrm{Ct}}$ method was adopted to calculate the relative expression level. The statistical difference among three biological duplicates, determined with one-way ANOVA, is shown via graphpad prism. The level of statistical significance was set at ns, $p>0.05,{ }^{*}, p<0.05 ;{ }^{* *}, p<0.01$; and $* * *, p<0.001$.

\subsection{Overexpression of BmTex261 Inhibited AcMNPV Infection in BmN Cells}

To further explore the function of BmTex261 in response to AcMNPV, the recombinant plasmid of pIZT-mCherry-BmTex261 was constructed (Figure 8A) and transfected into the $\mathrm{BmN}$ cells to overexpress BmTex261 (Figure 8B). The stable BmN cell line contained pIZT-mCherry-BmTex261 were generated by a final concentration of $200 \mu \mathrm{g} / \mathrm{mL}$ zeocin. The overexpression of BmTex 261 was detected by increasing about 56 -fold over the control group. Furthermore, the expression of lef3 of AcMNPV in the overexpression group was significantly decreased as compared to the control group at $24 \mathrm{~h}, 48 \mathrm{~h}$, and $72 \mathrm{~h}$ after AcMNPV infection (Figure 8C). 

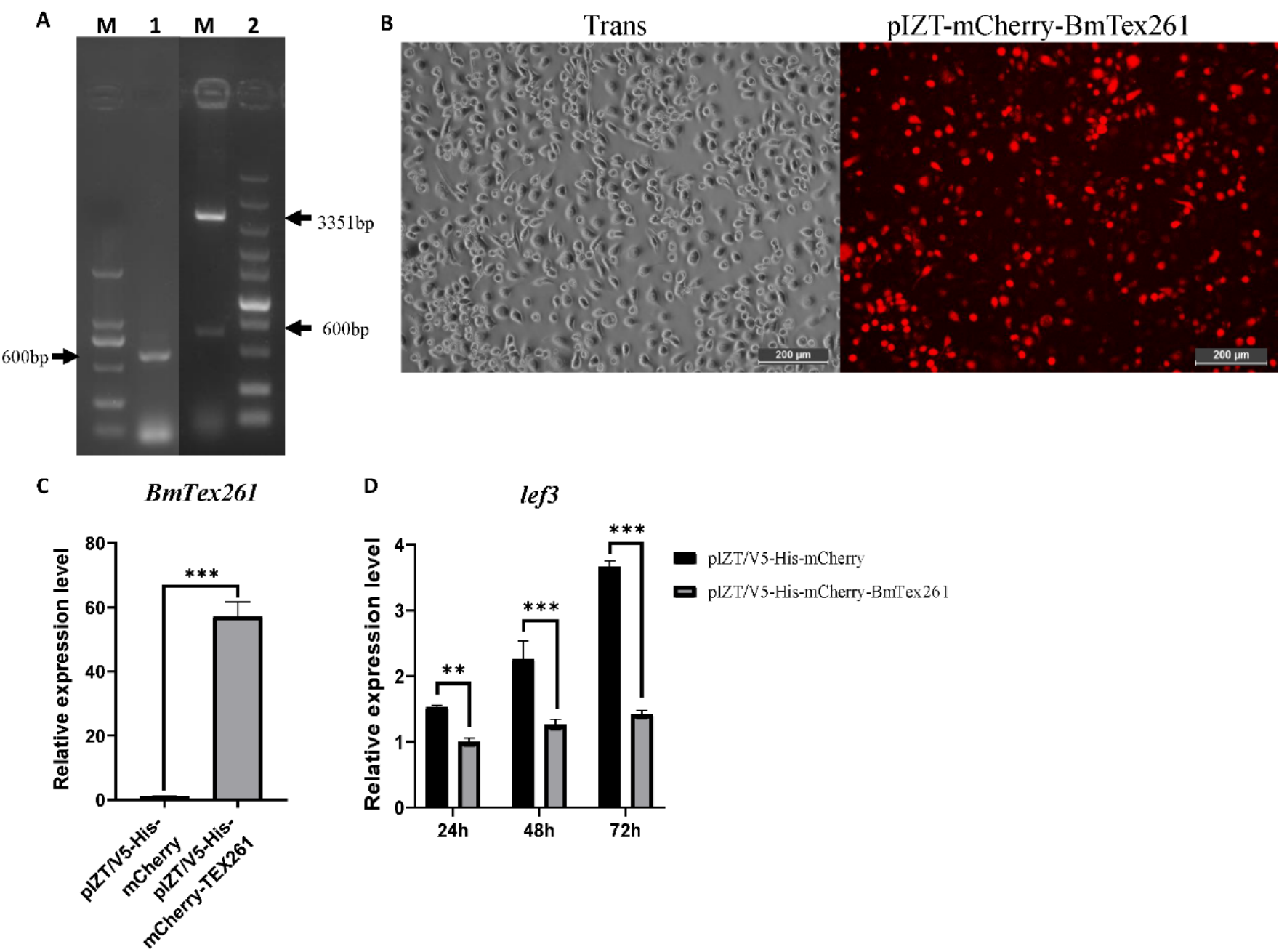

Figure 8. Analysis of AcMNPV infection after overexpression of BmTex261 at different durations in BmN cells. (A) Construction of pIZT-mCherry-BmTex261. (1) Amplification of CDS sequence of BmTex261 using PCR from BmN cells, the length of BmTex261 = 600 bp; (2) validation of the recombinant vector using Kpn I and Xba I, the length of BmTex261=600 bp, the length of the vector $=3351 \mathrm{bp} ;(\mathbf{B})$ overexpression detection of BmTex261 after transfection with pIZT-mCherry-BmTex261 in BmN cells. Red, mCherry; scale bar $=200 \mu \mathrm{m} ;(\mathbf{C})$ expression level analysis of BmTex261 in the stable cell line containing pIZT-mCherry-BmTex261; (D) analysis of lef3 expression after overexpression of BmTex261 at different durations. BmGAPDH was used to normalise the data that are shown as the mean \pm standard error; the mean was obtained from three independent repeats. The $2^{-\Delta \Delta C t}$ method was adopted to calculate the relative expression level. The statistical difference among three biological duplicates, determined with one-way ANOVA, is shown via graphpad prism. The level of statistical significance was set at ${ }^{* *}, p<0.01$; and ${ }^{* *}, p<0.001$.

\subsection{Knockdown BmTex261 Has No Effect on AcMNPV Infection in BmN Cells}

To knockdown BmTex261 at the BmN cell level, siRNAs were synthesised and transfected into BmN cells. Twenty-four hours was selected as the time point for analysis due to the best transfection efficiency, and AcMNPV was added at $24 \mathrm{~h}$ after transfection. The expression of BmTex261 was successfully knocked down at different durations after transfection with siRNAs in BmN cells (Figure 9A). To further verify the relationship between BmTex261 and AcMNPV, the expression level of lef3 at 24, 48, and $72 \mathrm{~h}$ after AcMNPV infection in the BmN cells was detected, and the results showed that knockdown of BmTex261 had no effect on AcMNPV infection (Figure 9B). 

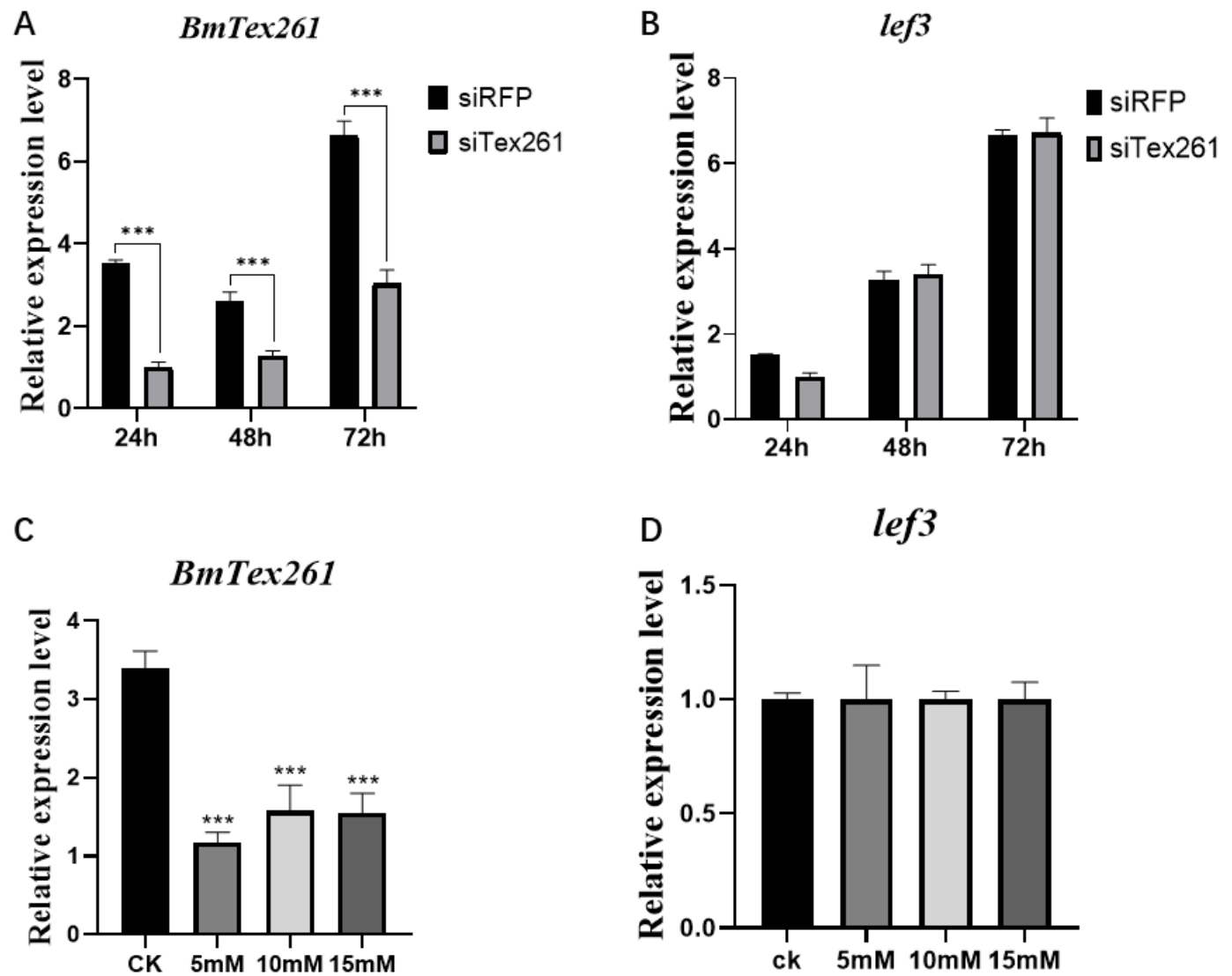

Figure 9. Analysis of AcMNPV infection after knocking down BmTex261 in BmN cells. (A) Expression analysis of BmTex261 after siTex261 transfection at different durations by using RT-qPCR. (B) Analysis of lef3 expression after knockdown of BmTex261 at different durations after AcMNPV infection. (C) Relative expression levels of BmTex261 after adding different concentrations of $\mathrm{CaCl}_{2} ;(\mathbf{D})$ relative expression levels of lef3 after adding different concentrations of $\mathrm{CaCl}_{2}$. BmGAPDH was used to normalise the data that are shown as the mean \pm standard error; the mean was obtained from three independent repeats. The $2^{-\Delta \Delta \mathrm{Ct}}$ method was adopted to calculate the relative expression level. The statistical difference among three biological duplicates, determined with one-way ANOVA, is shown via graphpad prism. The level of statistical significance was set at ${ }^{* * *}, p<0.001$.

Based on literature analysis, we found that Tex261 is related to $\mathrm{Ca}^{2+}[28,36,46]$. In this study, the different concentrations of $\mathrm{Ca}^{2+}$ were added to the medium; the final concentrations of $0,5,10$, and $15 \mathrm{mM}$ were used to analyse the function of BmTex261. The results showed that the expression of BmTex 261 was significantly downregulated after adding different concentrations of $\mathrm{Ca}^{2+}$ (Figure 9C). Moreover, the expression of lef3 was detected at $24 \mathrm{~h}$ after adding different concentrations of $\mathrm{CaCl}_{2}$. The expression level of lef3 was significantly different in this case (Figure 9D); this was consistent with the result after knockdown of BmTex261.

\section{Discussion}

BmNPV causes serious losses in silkworm production, and the underlying defence mechanism is still unclear. The AcMNPV is another representative baculovirus in insects and is largely similar to BmNPV. Normally, both viruses have strict host domains and generally do not cross-infect other host domains, but studies have found that recombinant AcMNPV can infect silkworms by punctures. This provides new incentives for the study of the mechanism of insect responses to baculovirus infection. In this study, transcriptome sequencing of AcMNPV infection-related genes in the hemolymph of B. mori was used to analyse the mechanism of silkworm defence against AcMNPV infection, which will be beneficial for clarifying the mechanism of silkworm defence against BmNPV. 
In this study, there are two strains with different resistances. The susceptible strain p50 cannot pupate and dies, whereas the resistant strain showed no effect after being infected with recombinant AcMNPV on the first day of the fifth instar (Figure 1). Here, the GC content of the four libraries was about $50 \%$, and all Q30\% were equal to or greater than 93.27\% (Table 3), indicating that the transcriptome data are of good quality. Correlation analysis between gene expression ratios obtained from transcriptome data and RT-PCR shows that the expression quantity is credible. Thus, the transcriptome results can be used for further studies.

Analysis of transcriptome data revealed 678 DEGs in the p50+ vs. p50- group and 515 DEGs in the C108+ vs. C108- group (Table 4). All the DEGs were listed in Supplementary Tables S1 and S2. Among them, the upregulation and downregulation of DEGs were almost similar in p50+ vs. p50- (Table 4); however, only half as many downregulated DEGs were upregulated DEGs in C108+ vs. C108- (Table 4), suggesting that AcMNPV has different effects on the two strains. Based on GO enrichment analysis of DEGs (Figure 3), we found that in the biological process, DEGs were mainly enriched in binding activity, but there were different binding types. Some cellular components, such as vesicle, endosome, and biological regulation in molecular function, differed among the different resistant strains; they were upregulated in p50+ vs. p50 - and downregulated in C108+ vs. C108 (Figure 3). These results indicate that AcMNPV causes different responses in silkworms with different resistances. In addition, the trend of genes related to biological regulation in the two groups was opposite, confirming the phenomenon that AcMNPV infection of p50 inhibited pupation (Figure 1). The KEGG analysis showed that DEGs were mainly enriched in metabolic pathways; some DEGs were enriched in the apoptosis pathway (Table 5). The KOG annotation showed that the metabolism-related families in p50 were more than those in C108 (Figure 5). In addition, DEGs in p50 of translation, ribosomal structure, and biogenesis were significantly more than that in C108 (Figure 5). The results were consistent with those in GO and KEGG. Some antioxidant-related proteins were found in the tryptophan metabolic pathway, such as catalase, which acts as a barrier against viral infections [47]. In Aedes aegypti, viral resistance is closely related to catalase, which is upregulated after hemolymph intake to protect the body from viruses in the hemolymph [48]. Catalase was also studied in BmNPV, but it cannot stop the infection from occurring since a coevolutionary bond is maintained between the virus and the host in B. mori [47]. Therefore, catalase should be a related gene that affects the resistance of silkworms. In addition, glutathione S-transferases (GSTs) are believed to play a role in the detoxification of xenobiotics, the resistance to insect viruses and pesticides, intracellular transport, the biosynthesis of hormones, and the protection against oxidative stress [49].

Apoptosis has long been regarded as a defence mechanism against viral infection (Table 5). Many genes related to regulating cancer cells can regulate apoptosis in silkworms [50-53]. In the apoptotic pathway, ultraspiracle (USP) was also found, along with its downstream gene transcription factor (E74). Since they have a certain relationship with the growth and metamorphosis of silkworms [54,55], they might be related to the infection with AcMNPV. In previous studies, Tex261 has been reported to induce cytotoxic death and was found in the apoptosis pathway [36]. Moreover, among the identified apoptosis genes, there was no report about the relationship of Testis expressed gene 261 (Tex261) and virus; it is valuable to detect the role of BmTex261 in AcMNPV infection. The expression levels of BmTex261 were measured in different tissues and developmental stages of the p50 strain by RT-qPCR; expression of BmTex261 was highest in the hemolymph (Figure 6A), and its immune stress response was most typical in the hemolymph after AcMNPV infection (Figure 7). These results may explain why AcMNPV can only infect some silkworms via the hemolymph by a puncture. Moreover, BmTex261 had different expression levels following AcMNPV infection in p50, but no significant differences in the C108 midgut, fat body, and malpighian tubule were observed (Figure 7). This gene was speculated to be related to the infection of silkworms with AcMNPV. The upregulated expression levels of BmTex261 in the midgut of C108 (Figure 7) may be a sign that AcMNPV is suppressed in the 
hemolymph, and thus, the virus cannot invade other tissues. In addition, the expression level of BmTex261 was highest in the pupal stage (Figure 6B), indicating that this gene plays an important role in the metamorphosis of silkworms and that p50 could not pupate after AcMNPV infection. To further investigate the role of BmTex261 in AcMNPV, the gene was overexpressed in $\mathrm{BmN}$ cells using the pIZT overexpression vector (Figure $8 \mathrm{~A}, \mathrm{~B}$ ). Based on the results, BmTex 261 was successfully overexpressed at $36 \mathrm{~h}$ (Figure $8 \mathrm{C}$ ). At 24, 48, and $72 \mathrm{~h}$ after the addition of AcMNPV, the expression level of the virus was significantly inhibited (Figure 8D), which leads us to infer that AcMNPV may regulate the expression level of the gene after invading silkworm cells, which reduces the resistance of silkworms and makes it impossible for the body to successfully immunise against the virus. The BmTex261 was knocked down by siRNA in the BmN cell (Figure 9A), but the reduced expression did not affect the expression of AcMNPV (Figure 9B). This may be due to the compensatory effects of the organism. To further verify this result, we analysed the differentially expressed genes, Tex261 and Inositol 1,4,5-trisphosphate (Ins-1,4,5-P3), which might have a certain relationship with $\mathrm{Ca}^{2+}[36,56]$, and we therefore speculated that the calcium ion level in silkworm also had a certain change. Besides, $\mathrm{Ca}^{2+}$ can significantly knockdown BmTex261 (Figure 9C), and its effect on the virus is consistent with that of RNAi, suggesting that knockdown BmTex261 can induce compensatory effects in B. mori.

\section{Conclusions}

In this study, DEGs were generated from transcriptomes of differentially resistant silkworm strains following AcMNPV infection, and the result of KEGG showed that DEGs in p50 were concentrated in the metabolic pathway and apoptosis pathway. Moreover, $B m T e x 261$ belonging to the apoptosis pathway was further analysed by overexpression and RNAi, and we found that the overexpression of BmTex261 could knockdown AcMNPV infection in BmN cells. The results in this study provide the theoretical reference for clarifying the mechanism of the infection and replication process of baculovirus in silkworms.

Supplementary Materials: The following are available online at https:/ /www.mdpi.com/article/10 $.3390 / \mathrm{pr} 9081401 / \mathrm{s} 1$, Figure S1: principal component analysis of the transcriptome, Table S1: DEGs in p50 strain, Table S2: DEGs in C108 strain.

Author Contributions: Conceived and designed the experiments: X.-y.D. and X.-y.W. Performed the experiments: X.-y.D. and C.-x.Z. Analysed the data: X.-y.D., Y.-h.K., S.Q. Contributed reagents/ materials/analysis tools: M.-w.L. and X.S. Wrote the paper: X.-y.D. and X.-y.W. All authors have read and agreed to the published version of the manuscript.

Funding: This work was supported by the National Natural Science Foundation of China, 31772523, Postgraduate Research \& Practice Innovation Program of Jiangsu Province (KYCX21_3513).

Institutional Review Board Statement: Not applicable.

Informed Consent Statement: Not applicable.

Data Availability Statement: The data that support the findings of this study are openly available in the SRA database at https:/ / www.ncbi.nlm.nih.gov/sra, accessed on 2 July 2021, reference number SRR15247045-SRR15247052. The data that support the findings of this study are available in the Supplementary Materials of this article.

Conflicts of Interest: The authors declare no conflict of interest.

\section{References}

1. Nerome, K.; Yamaguchi, R.; Fuke, N.; Izzati, U.Z.; Maegawa, K.; Sugita, S.; Kawasaki, K.; Kuroda, K.; Nerome, R. Development of a Japanese encephalitis virus genotype V virus-like particle vaccine in silkworms. J. Gen. Virol. 2018, 99, 897-907. [CrossRef]

2. Dash, R.; Mandal, M.; Ghosh, S.K.; Kundu, S.C. Silk sericin protein of tropical tasar silkworm inhibits UVB-induced apoptosis in human skin keratinocytes. Mol. Cell. Biochem. 2008, 311, 111-119. [CrossRef]

3. Nourmohammadi, J.; Roshanfar, F.; Farokhi, M.; Nazarpak, M.H. Silk fibroin/kappa-carrageenan composite scaffolds with enhanced biomimetic mineralization for bone regeneration applications. Mater. Sci. Eng. C 2017, 76, 951-958. [CrossRef] [PubMed] 
4. Yang, M. Silk-based biomaterials. Microsc. Res. Tech. 2017, 80, 321-330. [CrossRef] [PubMed]

5. Blissard, G.W.; Rohrmann, G.F. Baculovirus diversity and molecular biology. Annu. Rev. Entomol. 1990, 35, 127-155. [CrossRef] [PubMed]

6. Mori, H. Transovarian transmission of a foreign gene in the silkworm, Bombyx mori, by Autographa californica nuclear polyhedrosis virus. Biotechnology 1995, 13, 1005-1007. [CrossRef]

7. Rahman, M.; Gopinathan, K.P. Systemic and in vitro infection process of Bombyx mori nucleopolyhedrovirus. Virus Res. 2004, 101, 109-118. [CrossRef]

8. Katsuma, S.; Mita, K.; Shimada, T. ERK- and JNK-Dependent Signaling Pathways Contribute to Bombyx mori Nucleopolyhedrovirus Infection. J. Virol. 2007, 81, 13700-13709. [CrossRef]

9. Rohrmann, G.F. Baculovirus Molecular Biology, 2rd ed.; Bethesda: Rockville, MD, USA, 2013.

10. Slack, J.M.; Lawrence, S. Evidence for proteolytic cleavage of the baculovirus occlusion-derived virion envelope protein P74. J. Gen. Virol. 2005, 86, 1637-1643. [CrossRef] [PubMed]

11. Sparks, W.O.; Harrison, R.L.; Bonning, B.C. Autographa californica multiple nucleopolyhedrovirus ODV-E56 is a per os infectivity factor, but is not essential for binding and fusion of occlusion-derived virus to the host midgut. Virology 2011, 409, 69-76. [CrossRef] [PubMed]

12. Ayres, M.D.; Howard, S.C.; Kuzio, J.; Lopez-Ferber, M.; Possee, R.D. The Complete DNA Sequence of Autographa californica Nuclear Polyhedrosis Virus. Virology 1994, 202, 586-605. [CrossRef] [PubMed]

13. Gomi, S.; Majima, K.; Maeda, S. Sequence analysis of the genome of Bombyx mori nucleopolyhedrovirus. J. Gen. Virol. 1999, 80, 1323-1337. [CrossRef] [PubMed]

14. Bao, Y.-Y.; Tang, X.-D.; Lv, Z.-Y.; Wang, X.; Tian, C.-H.; Xu, Y.P.; Zhang, C.-X. Gene expression profiling of resistant and susceptible Bombyx mori strains reveals nucleopolyhedrovirus-associated variations in host gene transcript levels. Genomics 2009, 94, 138-145. [CrossRef]

15. Xue, J.; Qiao, N.; Zhang, W.; Cheng, R.; Zhang, X.-Q.; Bao, Y.-Y.; Xu, Y.-P.; Gu, L.-Z.; Han, J.-D.J.; Zhang, C.-X. Dynamic Interactions between Bombyx mori Nucleopolyhedrovirus and Its Host Cells Revealed by Transcriptome Analysis. J. Virol. 2012, 86, 7345-7359. [CrossRef] [PubMed]

16. Yin, H. Comprehensive analysis of lncRNA-mRNA regulatory network in BmNPV infected cells treated with Hsp90 inhibitor. Mol. Immunol. 2020, 127, 230-237. [CrossRef] [PubMed]

17. Zhang, S. Analysis of lncRNA-mediated gene regulatory network of Bombyx mori in response to BmNPV infection. J. Invertebr. Pathol. 2020, 170, 107323. [CrossRef]

18. Matos, L.; Gonçalves, V.; Pinto, E.; Laranjeira, F.; Prata, M.J.; Jordan, P.; Desviat, L.R.; Pérez, B.; Alves, S. Functional analysis of splicing mutations in the IDS gene and the use of antisense oligonucleotides to exploit an alternative therapy for MPS II. Biochim. Biophys. Acta (BBA) Mol. Basis Dis. 2015, 1852, 2712-2721. [CrossRef]

19. Hu, X.; Zhu, M.; Wang, S.; Zhu, L.; Xue, R.; Cao, G.; Gong, C. Proteomics analysis of digestive juice from silkworm during Bombyx mori nucleopolyhedrovirus infection. Proteomics 2015, 15, 2691-2700. [CrossRef]

20. Kang, L.; Shi, H.; Liu, X.; Zhang, C.; Yao, Q.; Wang, Y.; Chang, C.; Shi, J.; Cao, J.; Kong, J.; et al. Arginine kinase is highly expressed in a resistant strain of silkworm (Bombyx mori, Lepidoptera): Implication of its role in resistance to Bombyx mori nucleopolyhedrovirus. Comp. Biochem. Physiol. Part B Biochem. Mol. Biol. 2011, 158, 230-234. [CrossRef]

21. Flipsen, J.; Martens, J.; Van Oers, M.; Vlak, J.; Van Lent, J. Passage of Autographa californica Nuclear Polyhedrosis Virus through the Midgut Epithelium of Spodoptera exigua Larvae. Virology 1995, 208, 328-335. [CrossRef]

22. Washburn, J.O.; Chan, E.Y.; Volkman, L.E.; Aumiller, J.J.; Jarvis, D.L. Early Synthesis of Budded Virus Envelope Fusion Protein GP64 Enhances Autographa californica Multicapsid Nucleopolyhedrovirus Virulence in Orally Infected Heliothis virescens. J. Virol. 2003, 77, 280-290. [CrossRef]

23. Si, Y.-H.; Fang, M.; Huang, Y.; Zheng, F.-L.; Li, T.; Hu, Z.; Wang, H.-Z. Construction and Characterization of aHelicoverpa armigeraNucleopolyhedrovirus Bacterial Artificial Chromosome with Deletion of Ecdysteroid UDP-Glucosyltransferase Gene. Biosci. Biotechnol. Biochem. 2007, 71, 2435-2441. [CrossRef] [PubMed]

24. Wang, L.; Kiuchi, T.; Fujii, T.; Daimon, T.; Li, M.; Banno, Y.; Kikuta, S.; Kikawada, T.; Katsuma, S.; Shimada, T. Mutation of a novel $\mathrm{ABC}$ transporter gene is responsible for the failure to incorporate uric acid in the epidermis of ok mutants of the silkworm, Bombyx mori. Insect Biochem. Mol. Biol. 2013, 43, 562-571. [CrossRef] [PubMed]

25. Chen, Y.; Li, M.; Islam, I.; You, L.; Wang, Y.; Li, Z.; Ling, L.; Zeng, B.; Xu, J.; Huang, Y.; et al. Allelic-specific expression in relation to Bombyx mori resistance to Bt toxin. Insect Biochem. Mol. Biol. 2014, 54, 53-60. [CrossRef] [PubMed]

26. Braunagel, S.C. Identification of BV/ODV-C42, an Autographa californica nucleopolyhedrovirus orf101-encoded structural protein detected in infected-cell complexes with ODV-EC27 and p78/83. J. Virol. 2001, 75, 12331-12338. [CrossRef] [PubMed]

27. Wang, Y. Autographa californica multiple nucleopolyhedrovirus nucleocapsid protein BV/ODV-C42 mediates the nuclear entry of P78/83. J. Virol. 2008, 82, 4554-4561. [CrossRef]

28. Bhatia, N.K.; Carrillo, E.; Durham, R.J.; Berka, V.; Jayaraman, V. Allosteric Changes in the NMDA Receptor Associated with Calcium-Dependent Inactivation. Biophys. J. 2020, 119, 2349-2359. [CrossRef]

29. Li, K.; Wang, Y.; Bai, H.; Wang, Q.; Song, J.; Zhou, Y.; Wu, C.; Chen, X. The Putative Pocket Protein Binding Site of Autographa californica Nucleopolyhedrovirus BV/ODV-C42 Is Required for Virus-Induced Nuclear Actin Polymerization. J. Virol. 2010, 84, 7857-7868. [CrossRef] 
30. Vieira, H.L.A.; Pereira, A.C.P.; Carrondo, M.J.T.; Alves, P. Catalase effect on cell death for the improvement of recombinant protein production in baculovirus-insect cell system. Bioprocess Biosyst. Eng. 2006, 29, 409-414. [CrossRef]

31. Hillyer, J.F. Insect immunology and hematopoiesis. Dev. Comp. Immunol. 2016, 58, 102-118. [CrossRef]

32. Miller, L.K. Baculovirus interaction with host apoptotic pathways. J. Cell Physiol. 1997, 173, 178-182. [CrossRef]

33. Clem, R.J.; Fechheimer, M.; Miller, L.K. Prevention of apoptosis by a baculovirus gene during infection of insect cells. Science 1991, 254, 1388-1390. [CrossRef]

34. Bump, N.J.; Hackett, M.; Hugunin, M.; Seshagiri, S.; Brady, K.; Chen, P.; Ferenz, C.; Franklin, S.; Ghayur, T.; Li, P.; et al. Inhibition of ICE family proteases by baculovirus antiapoptotic protein p35. Science 1995, 269, 1885-1888. [CrossRef]

35. Lopez-Fernandez, L.A.; Parraga, M.; del Mazo, J. Tex261, a novel gene presumably related but distinct from steroidogenic acute regulatory (StAR) gene, is regulated during the development of germ cells. Biochem. Biophys. Res. Commun. 1998, 242, 565-569. [CrossRef]

36. Taniura, H. Tex261 modulates the excitotoxic cell death induced by N-methyl-D-aspartate (NMDA) receptor activation. Biochem. Biophys. Res. Commun. 2007, 362, 1096-1100. [CrossRef] [PubMed]

37. Li, T. The haemolymph melanization response is related to defence against the AcMNPV infection in Bombyx mori. Arch. Insect Biochem. Physiol. 2021, 2021, e21764.

38. Chen, S.; Zhou, Y.; Chen, Y.; Gu, J. fastp: An ultra-fast all-in-one FASTQ preprocessor. Bioinformatics 2018, 34, i884-i890. [CrossRef]

39. The International Silkworm Genome Consortium. The genome of a lepidopteran model insect, the silkworm Bombyx mori. Insect Biochem. Mol. Biol. 2008, 38, 1036-1045. [CrossRef] [PubMed]

40. Mita, K.; Kasahara, M.; Sasaki, S.; Nagayasu, Y.; Yamada, T.; Kanamori, H.; Namiki, N.; Kitagawa, M.; Yamashita, H.; Yasukochi, Y.; et al. The Genome Sequence of Silkworm, Bombyx mori. DNA Res. 2004, 11, 27-35. [CrossRef] [PubMed]

41. Biology Analysis Group; Xia, Q.; Zhou, Z.; Lu, C.; Cheng, D.; Dai, F.-Y.; Liu, B.; Zhao, P.; Zha, X.; Cheng, T.; et al. A Draft Sequence for the Genome of the Domesticated Silkworm (Bombyx mori). Science 2004, 306, 1937-1940. [CrossRef]

42. Kim, D.; Paggi, J.M.; Park, C.; Bennett, C.; Salzberg, S.L. Graph-based genome alignment and genotyping with HISAT2 and HISAT-genotype. Nat. Biotechnol. 2019, 37, 907-915. [CrossRef] [PubMed]

43. Pertea, M.; Kim, D.; Pertea, G.M.; Leek, J.T.; Salzberg, M.P.D.K.G.M.P.S.L. Transcript-level expression analysis of RNA-seq experiments with HISAT, StringTie and Ballgown. Nat. Protoc. 2016, 11, 1650-1667. [CrossRef]

44. Tatusov, R.L.; Natale, D.A.; Garkavtsev, I.V.; Tatusova, T.A.; Shankavaram, U.T.; Rao, B.S.; Kiryutin, B.; Galperin, M.; Fedorova, N.D.; Koonin, E.V. The COG database: New developments in phylogenetic classification of proteins from complete genomes. Nucleic Acids Res. 2001, 29, 22-28. [CrossRef]

45. Tatusov, R.L.; Fedorova, N.D.; Jackson, J.D.; Jacobs, A.R.; Kiryutin, B.; Koonin, E.V.; Krylov, D.M.; Mazumder, R.; Mekhedov, S.L.; Nikolskaya, A.N.; et al. The COG database: An updated version includes eukaryotes. BMC Bioinform. 2003, 4, 41. [CrossRef] [PubMed]

46. Puri, B.K. Calcium Signaling and Gene Expression. Adv. Exp. Med. Biol. 2019, 1131, 537-545.

47. Vessaro-Silva, A.S.; Neto, M.H.M.; Brancalhão, R.M.C.; Ribeiro, L.D.F.C.; Guimarães, A.T.B.; De Oliveira, C.M.T. Antioxidant Systems as a Response to Midgut Cellular of Bombyx mori Lineu, 1758 (Lepidoptera: Bombycidae) Infection for Baculoviruses. J. Econ. Èntomol. 2019, 112, 1089-1097. [CrossRef]

48. Oliveira, J.H.M.; Talyuli, O.A.C.; Goncalves, R.L.S.; Paiva-Silva, G.O.; Sorgine, M.H.F.; Alvarenga, P.H.; Oliveira, P. Catalase protects Aedes aegypti from oxidative stress and increases midgut infection prevalence of Dengue but not Zika. PLOS Neglected Trop. Dis. 2017, 11, e0005525. [CrossRef] [PubMed]

49. Zou, F.-M.; Lou, D.-S.; Zhu, Y.-H.; Wang, S.-P.; Jin, B.-R.; Gui, Z.-Z. Expression profiles of glutathione S-transferase genes in larval midgut of Bombyx mori exposed to insect hormones. Mol. Biol. Rep. 2010, 38, 639-647. [CrossRef]

50. Zhuang, W.; Zhang, C.; Hao, F.; Sun, X. Baculoviral IAP Repeat Containing 6 (BIRC6) Is a Predictor of Prognosis in Prostate Cancer. Med Sci. Monit. 2018, 24, 839-845. [CrossRef]

51. Orgueira, A.M.; López, M.C.; Raíndo, A.P.; Arias, J.D.; Rodríguez, B.A.; Pérez, L.B.; Vence, N.A.; Ángeles, B.L.; Blanco, A.A.; Valentín, P.M.; et al. Detection of Rare Germline Variants in the Genomes of Patients with B-Cell Neoplasms. Cancers 2021, 13, 1340. [CrossRef]

52. Xiong, C.; Wang, Z.; Wang, G.; Zhang, C.; Jin, S.; Jiang, G.; Bai, D. Identification of CDC20 as an immune infiltration-correlated prognostic biomarker in hepatocellular carcinoma. Investig. New Drugs 2021, 1-15. [CrossRef]

53. Wang, L.; Wang, M.; Hu, C.; Li, P.; Qiao, Y.; Xia, Y.; Liu, L.; Jiang, X. Protein salvador homolog 1 acts as a tumor suppressor and is modulated by hypermethylation in pancreatic ductal adenocarcinoma. Oncotarget 2017, 8, 62953-62961. [CrossRef] [PubMed]

54. Swevers, L.; Cherbas, L.; Cherbas, P.; Iatrou, K. Bombyx EcR (BmEcR) and Bombyx USP (BmCF1) combine to form a functional ecdysone receptor. Insect Biochem. Mol. Biol. 1996, 26, 217-221. [CrossRef]

55. Yao, T.-P.; Segraves, W.A.; Oro, A.E.; McKeown, M.; Evans, R. Drosophila ultraspiracle modulates ecdysone receptor function via heterodimer formation. Cell 1992, 71, 63-72. [CrossRef]

56. Ryu, S.H.; Lee, S.Y.; Lee, K.; Rhee, S.G. Catalytic properties of inositol trisphosphate kinase: Activation by Ca ${ }^{2+}$ and calmodulin. FASEB J. 1987, 1, 388-393. [CrossRef] [PubMed] 